\title{
Erratum to: Search for squarks and gluinos in final states with one isolated lepton, jets, and missing transverse momentum at $\sqrt{s}=13 \mathrm{TeV}$ with the ATLAS detector
}

\section{ATLAS Collaboration ${ }^{\star}$}

CERN, 1211 Geneva 23, Switzerland

Published online: 29 October 2021

(C) CERN for the benefit of the ATLAS collaboration 2021

Erratum to: Eur. Phys. J. C 81:600 (2021)

https://doi.org/10.1140/epjc/s10052-021-09344-w

In this article the title was incorrectly given as

'Search for squarks and gluinos in final states with one isolated lepton, jets, and missing transverse momentum at $\sqrt{s}=13$ with the ATLAS detector' but should have been

'Search for squarks and gluinos in final states with one isolated lepton, jets, and missing transverse momentum at $\sqrt{s}=13 \mathrm{TeV}$ with the ATLAS detector'.

The original article has been corrected.
Open Access This article is licensed under a Creative Commons Attribution 4.0 International License, which permits use, sharing, adaptation, distribution and reproduction in any medium or format, as long as you give appropriate credit to the original author(s) and the source, provide a link to the Creative Commons licence, and indicate if changes were made. The images or other third party material in this article are included in the article's Creative Commons licence, unless indicated otherwise in a credit line to the material. If material is not included in the article's Creative Commons licence and your intended use is not permitted by statutory regulation or exceeds the permitted use, you will need to obtain permission directly from the copyright holder. To view a copy of this licence, visit http://creativecomm ons.org/licenses/by/4.0/.

Funded by $\mathrm{SCOAP}^{3}$.
The original article can be found online at https://doi.org/10.1140/ epjc/s10052-021-09344-w. 


\section{ATLAS Collaboration}

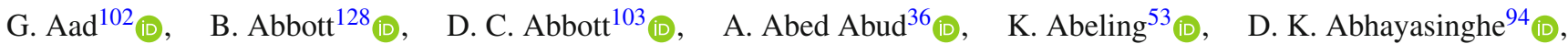
S. H. Abidi ${ }^{167}$ (D), O. S. AbouZeid ${ }^{40}$ (D), N. L. Abraham ${ }^{156}, \quad$ H. Abramowicz ${ }^{161}$ (D), H. Abreu ${ }^{160}$ (D), Y. Abulaiti ${ }^{6}$ (D),

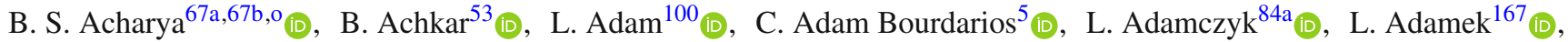

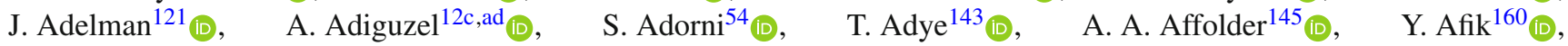
C. Agapopoulou ${ }^{65}$ (D) M. N. Agaras $^{38}$ (D), A. Aggarwal ${ }^{119}$ (D), C. Agheorghiesei ${ }^{27 c}$ (D), J. A. Aguilar-Saavedra ${ }^{139 a, 139 f, a c}$ (D),

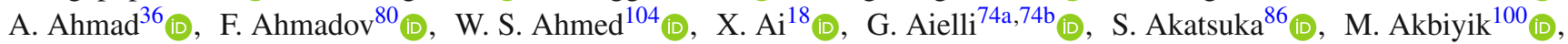

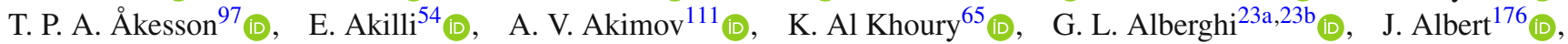

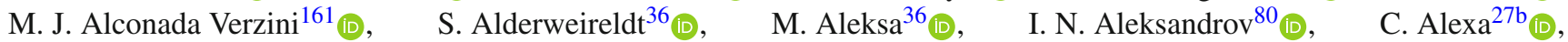

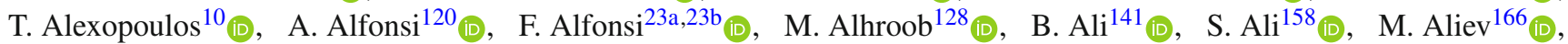

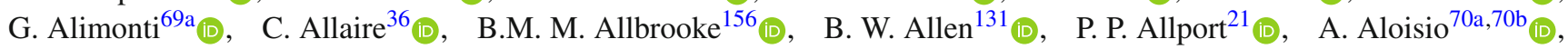

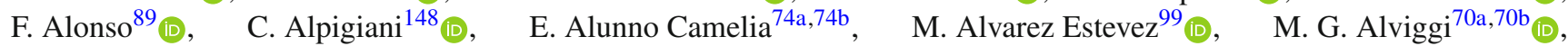
Y. Amaral Coutinho ${ }^{81 b}$ (D) A. Ambler ${ }^{104}$ (D) , L. Ambroz ${ }^{134}$ (D) , C. Amelung ${ }^{36}$, D. Amidei ${ }^{106}$ (D) , S. P. Amor Dos Santos ${ }^{139 a}$ (D),

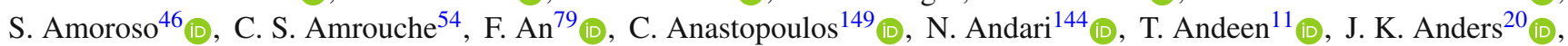

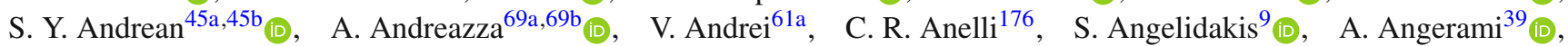

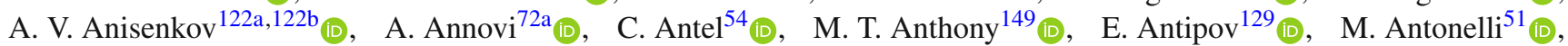

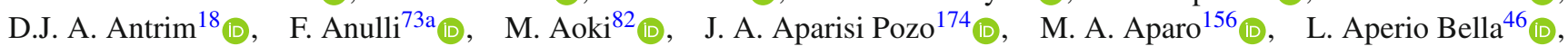

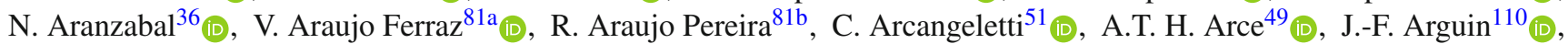

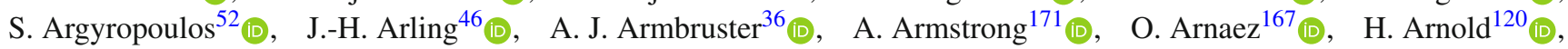

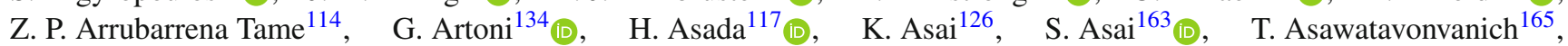

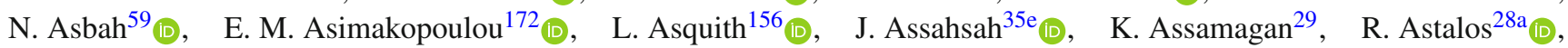

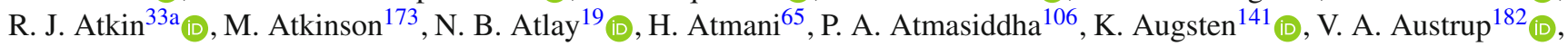

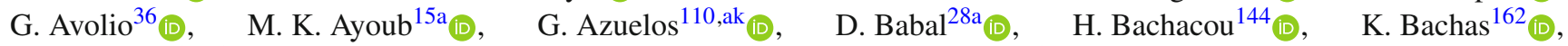

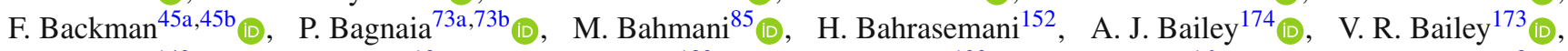

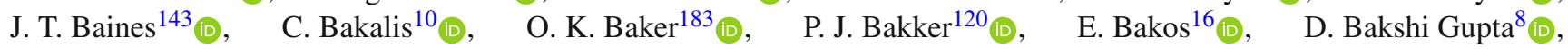
S. Balaji ${ }^{157}$ (D), R. Balasubramanian ${ }^{120}$ (D), E. M. Baldin ${ }^{122 a, 122 b}$ (D) $^{2}$ P. Balek ${ }^{180}$ (D), F. Balli ${ }^{144}$ (D), W. K. Balunas ${ }^{134}$ (D), J. Balz ${ }^{100}$ (D), E. Banas ${ }^{85}$ (D), M. Bandieramonte ${ }^{138}$ (D), A. Bandyopadhyay ${ }^{19}$ (D), Sw. Banerjee ${ }^{181, j}$ (D), L. Barak ${ }^{161}$ (D),

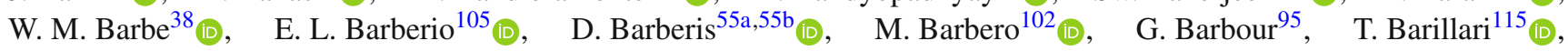

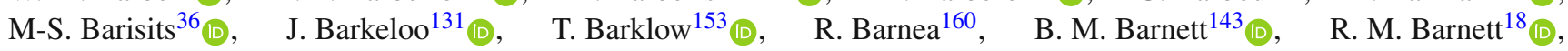

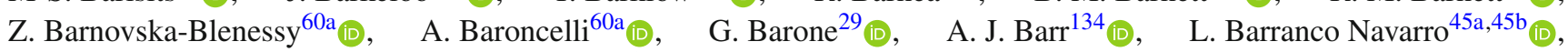

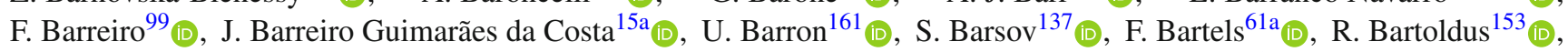

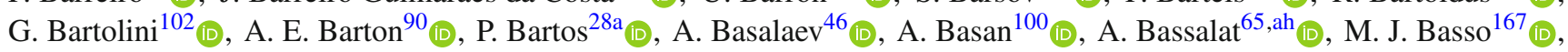

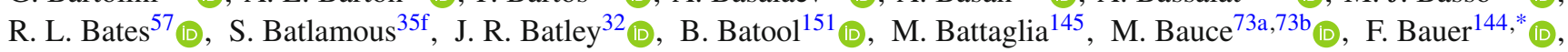

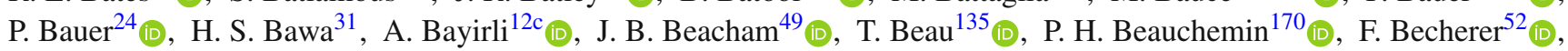

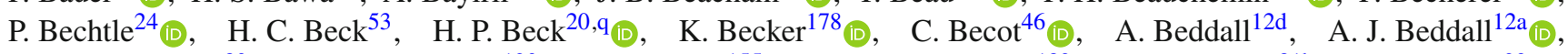

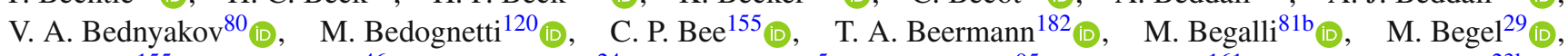

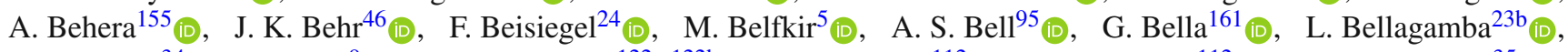

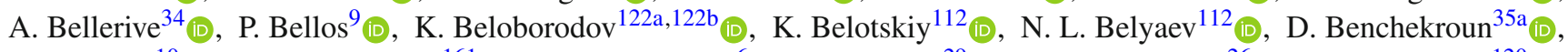

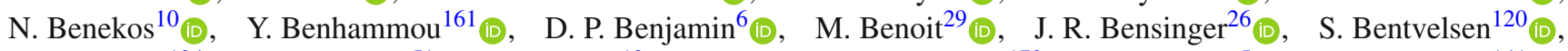
L. Beresford ${ }^{134}$ (I), M. Beretta ${ }^{51}$ (D), D. Berge ${ }^{19}$ (D), E. Bergeaas Kuutmann ${ }^{172}$ (I), N. Berger ${ }^{5}$ (D), B. Bergmann ${ }^{141}$ (D),

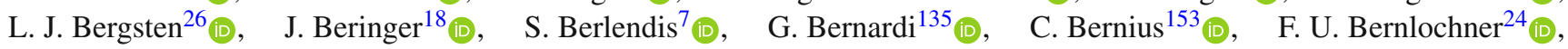
T. Berry ${ }^{94}$ (D), P. Berta ${ }^{100}{ }^{(D)}, \quad$ A. Berthold ${ }^{48}$ (D), I. A. Bertram ${ }^{90}$ (D), O. Bessidskaia Bylund ${ }^{182}$ (D), N. Besson ${ }^{144}$ (D), S. Bethke ${ }^{115}$ (D), A. Betti ${ }^{42}$ (I), A. J. Bevan ${ }^{93}$ (D), J. Beyer ${ }^{115}$ (D), S. Bhatta ${ }^{155}$ (D), D. S. Bhattacharya ${ }^{177}$ (I), P. Bhattarai ${ }^{26}$,

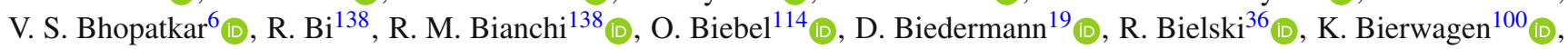

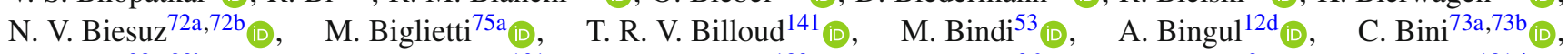

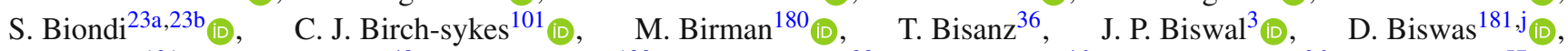

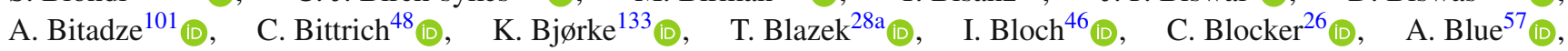

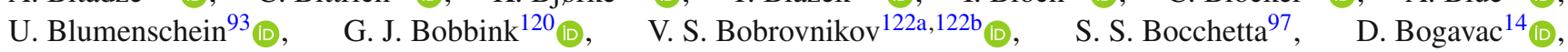

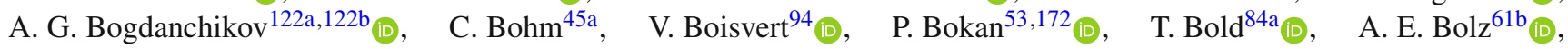
M. Bomben ${ }^{135}$ (D), M. Bona ${ }^{93}$ (D), J. S. Bonilla ${ }^{131}$ (D), M. Boonekamp ${ }^{144}$ (D), C. D. Booth ${ }^{94}$ (D), A. G. Borbély ${ }^{57}$ (D),

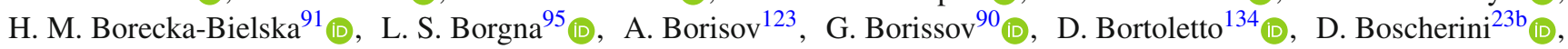
M. Bosman ${ }^{14}$ (i), J. D. Bossio Sola ${ }^{104}$ (i), K. Bouaouda ${ }^{\left.35 a_{(}\right)} \quad$ J. Boudreau $^{138}$ (i), E. V. Bouhova-Thacker ${ }^{90}$ (D), 


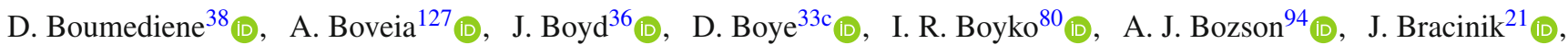

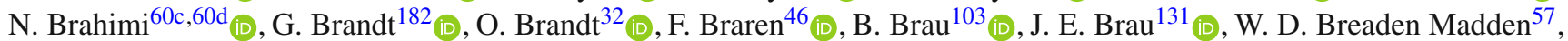

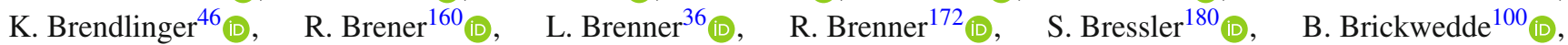

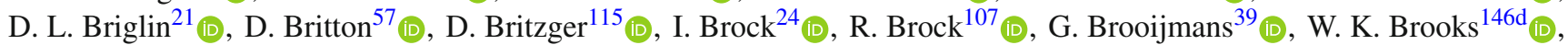

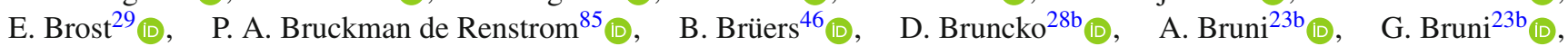

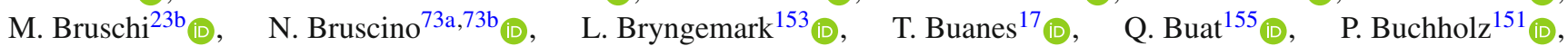
A. G. Buckley ${ }^{57}$ (D), I. A. Budagov ${ }^{80}$ (D), M. K. Bugge ${ }^{133}$ (D), O. Bulekov ${ }^{112}$ (D), B. A. Bullard ${ }^{59}$ (D), T. J. Burch ${ }^{121}$ (D),

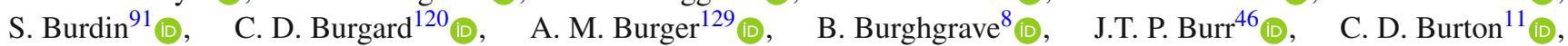

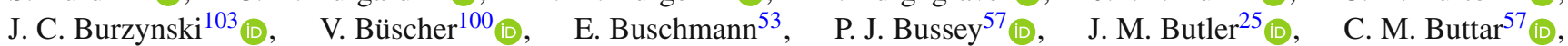
J. M. Butterworth ${ }^{95}$ (D), P. Butti ${ }^{36}$, W. Buttinger ${ }^{143}$ (D), C. J. Buxo Vazquez ${ }^{107}$, A. Buzatu ${ }^{158}$ (D), A. R. Buzykaev ${ }^{122 a, 122 b}$ (D), $^{\text {(D) }}$ G. Cabras ${ }^{23 a}, 23 b$ (D), S. Cabrera Urbán ${ }^{174}$ (D), D. Caforio ${ }^{56}$ (D) H. Cai ${ }^{138}$ (D), V. M. M. Cairo ${ }^{153}$ (D), O. Cakir ${ }^{4 a}$ (D), N. Calace ${ }^{36}$ (D) P. Calafiura ${ }^{18}$ (D) G. Calderini ${ }^{135}$ (D) P. Calfayan ${ }^{66}$ (D) , G. Callea ${ }^{57}$ (D) L. P. Caloba ${ }^{81 b}$, A. Caltabiano ${ }^{74 a, 74 b}$, S. Calvente Lopez ${ }^{99}$ (D), D. Calvet ${ }^{38}$ (D), S. Calvet ${ }^{38}$ (D), T. P. Calvet ${ }^{102}{ }_{(\mathbb{D})}$, M. Calvetti ${ }^{72 a, 72 b}$ (D), R. Camacho Toro ${ }^{135}{ }_{(\mathbb{D})}$,

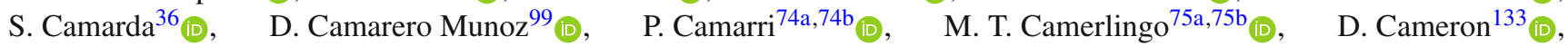

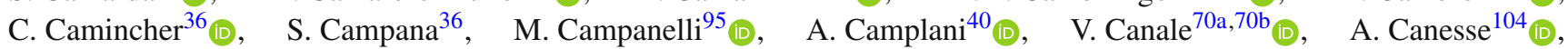

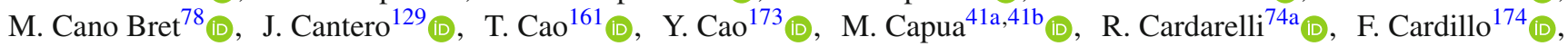

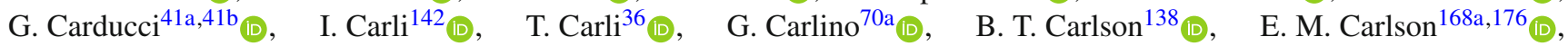

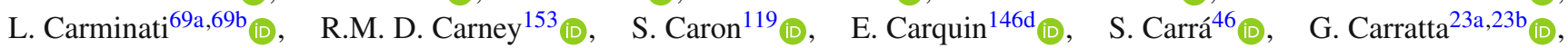

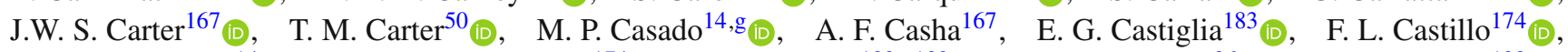
L. Castillo Garcia ${ }^{14}$ (D), V. Castillo Gimenez ${ }^{174}$ (D), N. F. Castro ${ }^{139 a, 139 e} e_{\mathbb{C}}, \quad$ A. Catinaccio $^{36}$ (D), J. R. Catmore ${ }^{133}$ (D),

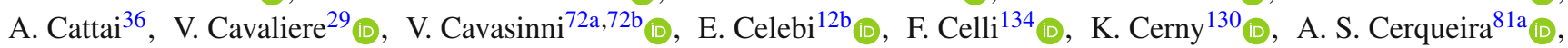
A. Cerri ${ }^{156}$ (D) L. Cerrito ${ }^{74 a, 74 b}$ (D) F. Cerutti ${ }^{18}$ (D) A. Cervelli ${ }^{23 a, 23 b}{ }_{(D)}$, S. A. Cetin ${ }^{12 b}$ (D) Z. Chadi ${ }^{35 a}$, D. Chakraborty ${ }^{121}$ (D), J. Chan ${ }^{181}$ (D), W. S. Chan ${ }^{120}$ (D), W. Y. Chan ${ }^{91}$ (D), J. D. Chapman ${ }^{32}$ (D) $\quad$ B. Chargeishvili ${ }^{159 b}$ (D), D. G. Charlton ${ }^{21}$ (D),

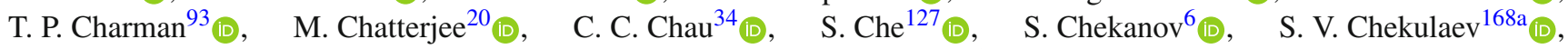

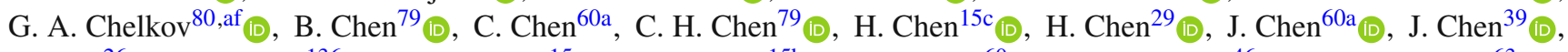

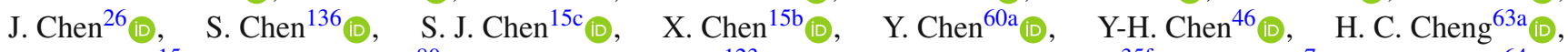
H. J. Cheng ${ }^{15 a}$ (D) A. Cheplakov ${ }^{80}$ (D) E. Cheremushkina ${ }^{123}$ (D) R. Cherkaoui El Moursli ${ }^{35 f}$ (ID, E. Cheu ${ }^{7}$, K. K. Cheung ${ }^{64}$ (D),

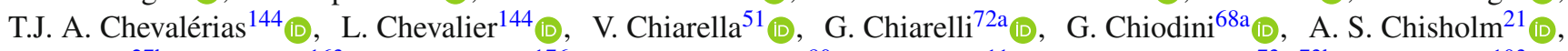

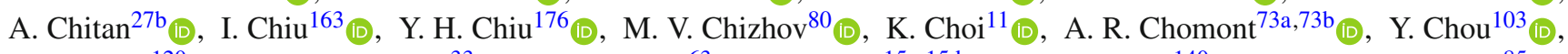

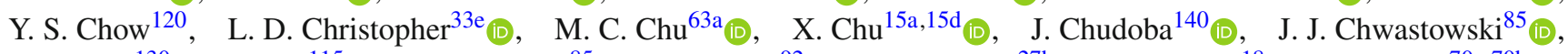

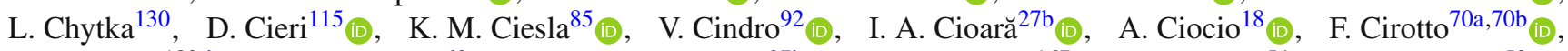

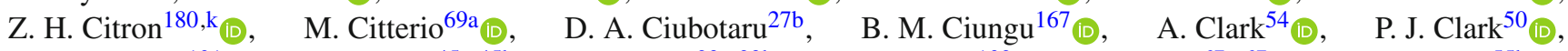

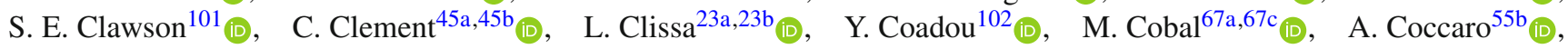
J. Cochran ${ }^{79}$, R. Coelho Lopes De $\mathrm{Sa}^{103}$ (I),$\quad$ H. Cohen ${ }^{161}, \quad$ A. E. C. Coimbra ${ }^{36}$ (I), B. Cole ${ }^{39}$ (D), A. P. Colijn ${ }^{120}$,

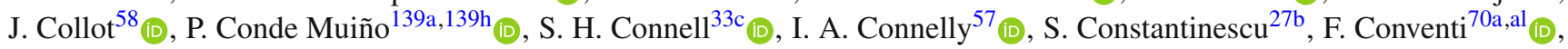

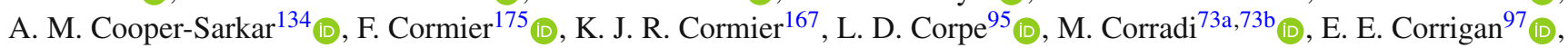

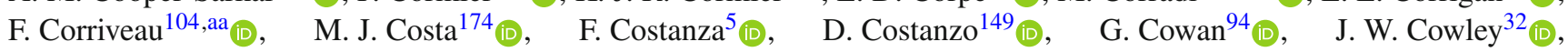
J. Crane ${ }^{101}$ (D) K. Kranmer ${ }^{125}$ (D), R. A. Creager ${ }^{136}$ (D), S. Crépé-Renaudin ${ }^{58}$ (D) F. Crescioli ${ }^{135}$ (D), M. Cristinziani ${ }^{24}$ (D),

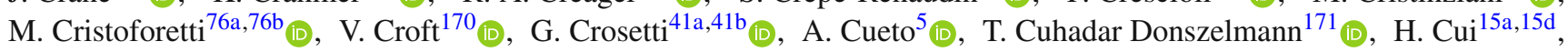

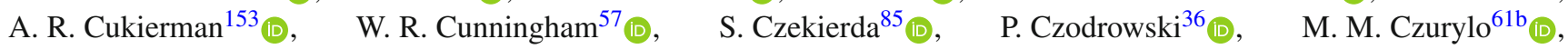

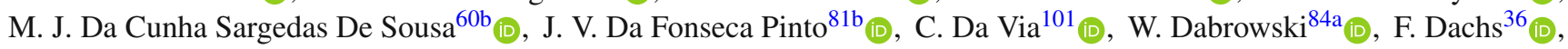

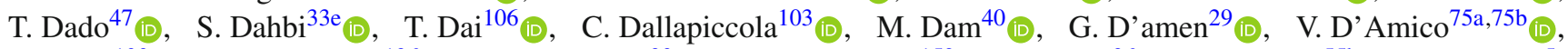

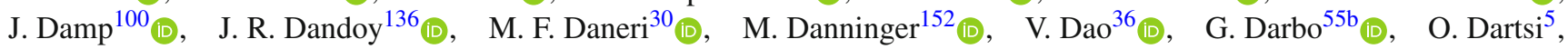

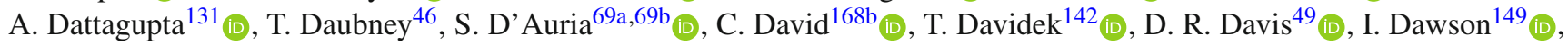

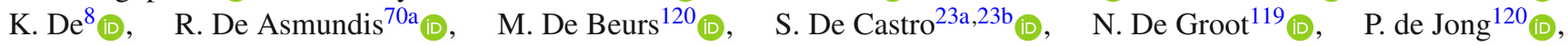

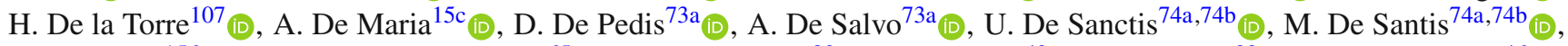

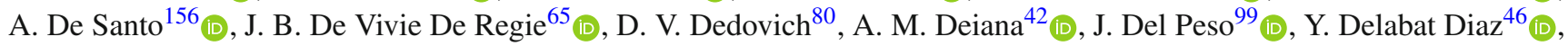

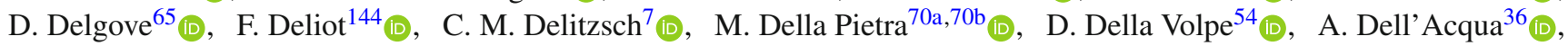

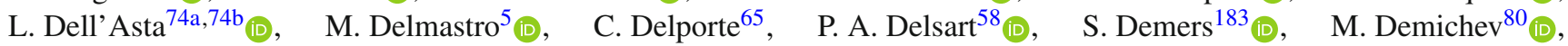

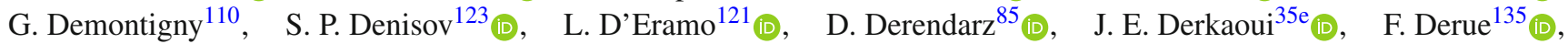

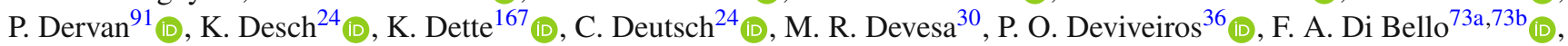

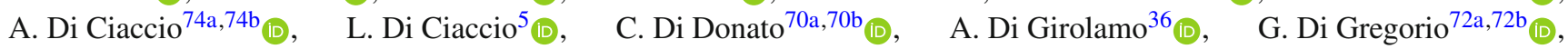

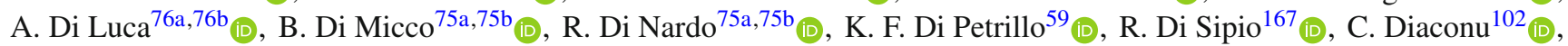




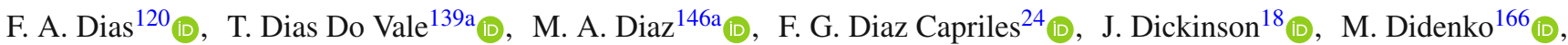

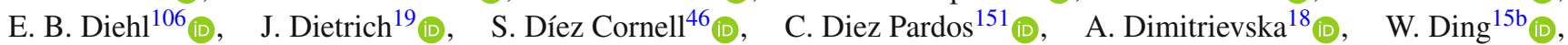

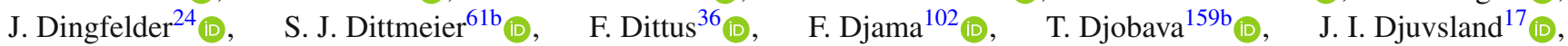
M.A. B. Do Vale ${ }^{147}$ (D), M. Dobre ${ }^{27 b}$ (D),$\quad$ D. Dodsworth ${ }^{26}$ (D) $\quad$ C. Doglioni ${ }^{97}$ (D), J. Dolejsi ${ }^{142}$ (D), Z. Dolezal ${ }^{142}$ (D),

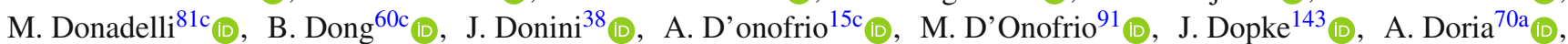

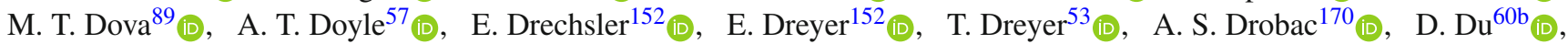

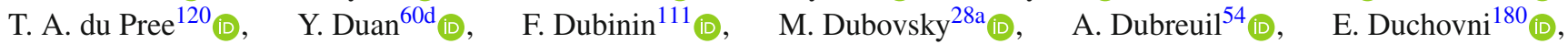

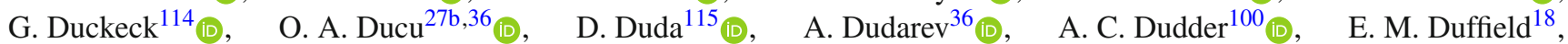

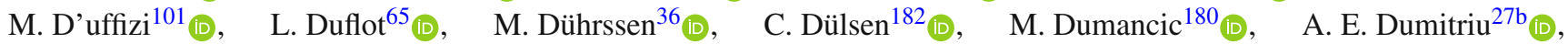

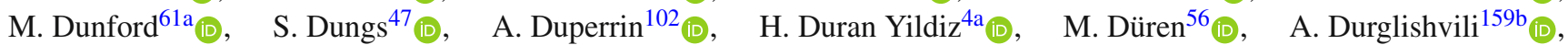

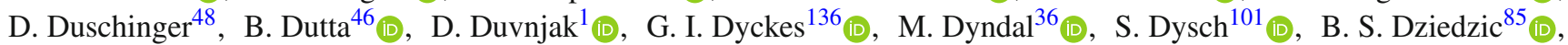
M. G. Eggleston ${ }^{49}$, T. Eifert $^{8}$ (D), G. Eigen $^{17}$ (D), K. Einsweiler ${ }^{18}$ (D), T. Ekelof ${ }^{172}$ (D) H. El Jarrari ${ }^{35 f}$ (D), V. Ellajosyula ${ }^{172}$ (D), M. Ellert ${ }^{172}$ (D) , F. Ellinghaus ${ }^{182}$ (D), A. A. Elliot $^{93}$ (D), N. Ellis $^{36}$ (D) J. Elmsheuser ${ }^{29}$ (D) M. Elsing ${ }^{36}$ (D), D. Emeliyanov ${ }^{143}$ (D),

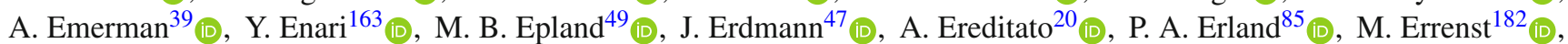

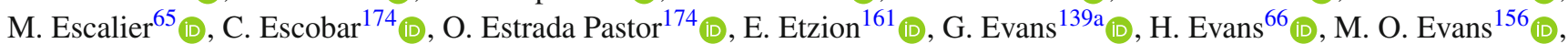

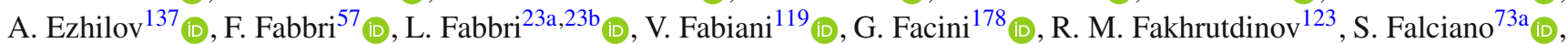

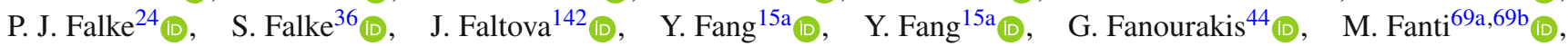

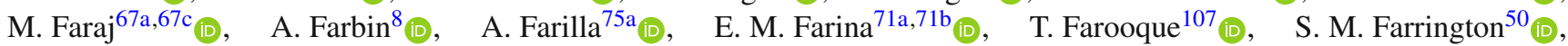
P. Farthouat ${ }^{36}$ (D), F. Fassi ${ }^{35 f}$ (I), P. Fassnacht ${ }^{36}$ (D), D. Fassouliotis ${ }^{9}$ (D), M. Faucci Giannelli ${ }^{50}$ (D), W. J. Fawcett ${ }^{32}$ (D),

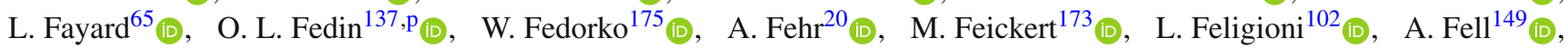
C. Feng ${ }^{60 b}{ }_{(\mathbb{D})}$, M. Feng $^{49}$ (D), M. J. Fenton ${ }^{171}$ (D), A. B. Fenyuk ${ }^{123}$, S. W. Ferguson ${ }^{43}$ (D), J. Ferrando ${ }^{46}$ (D), A. Ferrari ${ }^{172}$ (D),

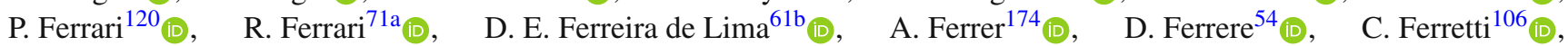

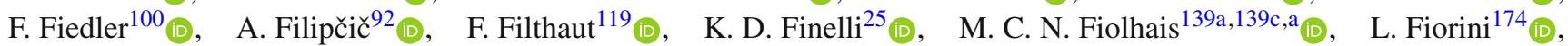

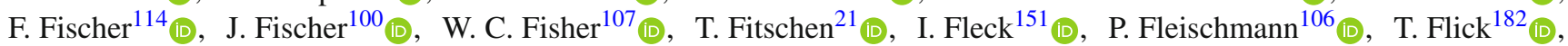

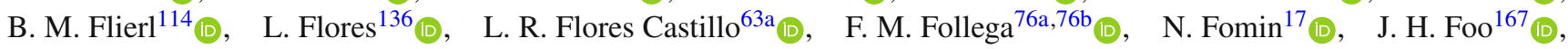

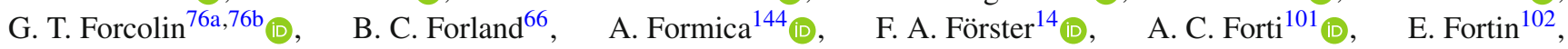

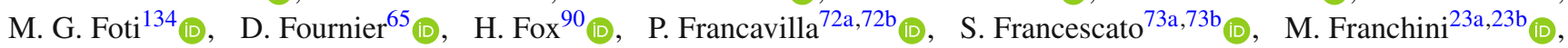

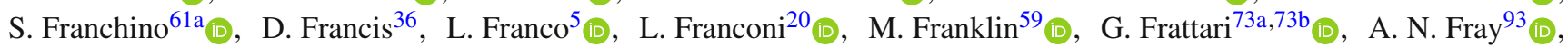

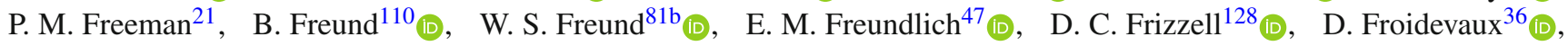
J. A. Frost ${ }^{134}\left(\mathbb{D}, \quad\right.$ M. Fujimoto ${ }^{126}$ (D), C. Fukunaga ${ }^{164}$ (D), E. Fullana Torregrosa ${ }^{174}$ (D), T. Fusayasu ${ }^{116}, \quad$ J. Fuster ${ }^{174}$ (D), A. Gabrielli ${ }^{23 a, 23 b}{ }_{(\mathbb{D}}, \quad$ A. Gabrielli ${ }^{36}$ (D), S. Gadatsch ${ }^{54}$ (D), P. Gadow ${ }^{115}$ (D), G. Gagliardi ${ }^{55 a, 55 b}$ (D), L. G. Gagnon ${ }^{110}{ }_{(\mathbb{D})}$, G. E. Gallardo ${ }^{134}$ (D), E. J. Gallas ${ }^{134}$ (I),$\quad$ B. J. Gallop ${ }^{143}$ (D), R. Gamboa Goni ${ }^{93}$ (I), K. K. Gan ${ }^{127}$ (D), S. Ganguly ${ }^{180}$ (D),

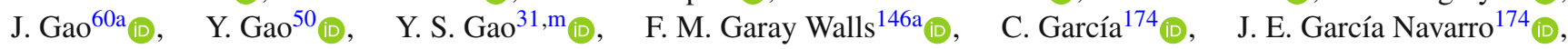

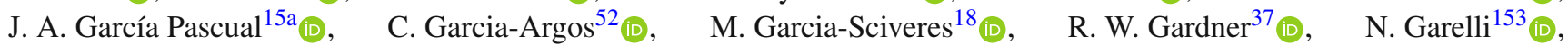

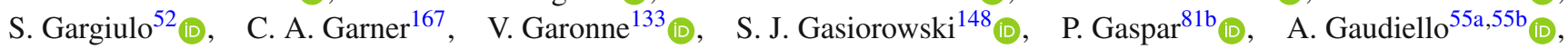

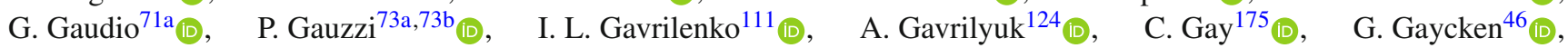

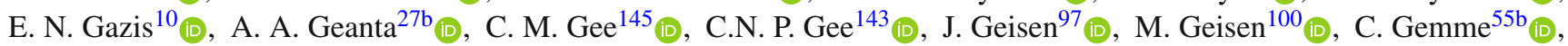

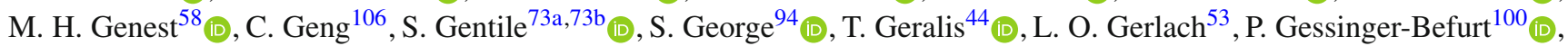

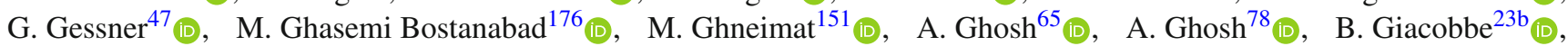

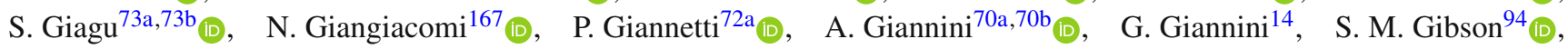

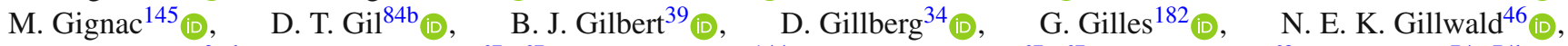

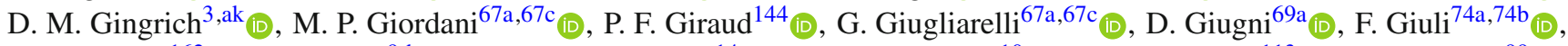

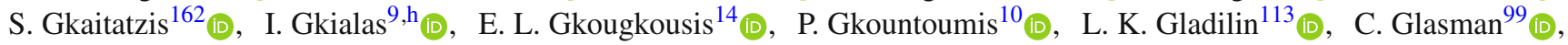

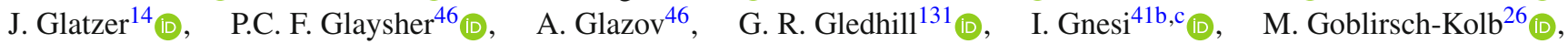
D. Godin ${ }^{110}, \quad$ S. Goldfarb ${ }^{105}$ (D),$\quad$ T. Golling ${ }^{54}$ (D) , D. Golubkov ${ }^{123}$ (D), A. Gomes ${ }^{139 a}, 139 b$ (D), R. Goncalves Gama ${ }^{53}$ (D),

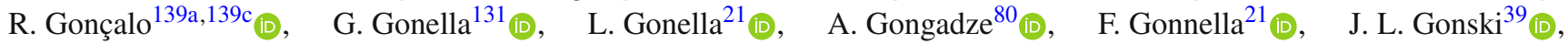
S. González de la Hoz ${ }^{174}$ (D), S. Gonzalez Fernandez ${ }^{14}$ (D), R. Gonzalez Lopez ${ }^{91}$ (D), $\quad$ C. Gonzalez Renteria ${ }^{18}$ (D), R. Gonzalez Suarez ${ }^{172}$ (I) S. Sonzalez-Sevilla ${ }^{54}$ (D), G. R. Gonzalvo Rodriguez ${ }^{174}$ (D), L. Goossens ${ }^{36}$ (D), N. A. Gorasia ${ }^{21}$ (D), P. A. Gorbounov ${ }^{124}$ (D), H. A. Gordon ${ }^{29}$ (D), B. Gorini ${ }^{36}$ (D), E. Gorini ${ }^{68 a}, 68 b$ (D), A. Gorišek ${ }^{92}$ (D), A. T. Goshaw ${ }^{49}$ (D),

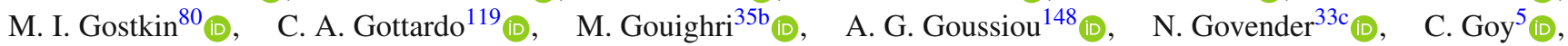

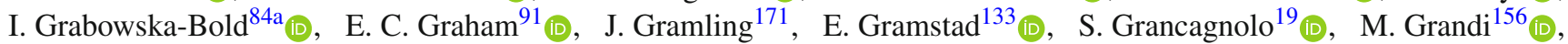

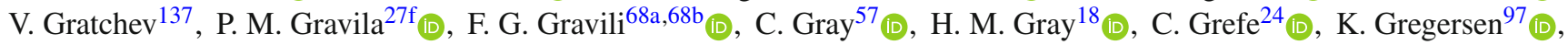

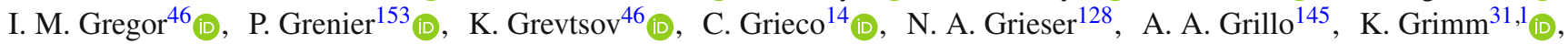




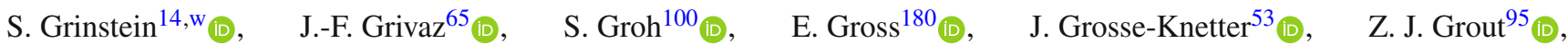
C. Grud ${ }^{106}$, A. Grummer ${ }^{118}$ (D), J. C. Grundy ${ }^{134}$ (D), L. Guan ${ }^{106}$ (D), W. Guan ${ }^{181}$ (D), C. Gubbels ${ }^{175}$ (D), J. Guenther ${ }^{77}$ (D), A. Guerguichon ${ }^{65}$ (D), J.G. R. Guerrero Rojas ${ }^{174}$ (D), F. Guescini ${ }^{115}$ (D), D. Guest ${ }^{77}$ (D), R. Gugel ${ }^{100}$ (D), A. Guida $^{46}$ (D),

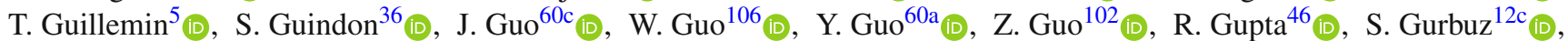

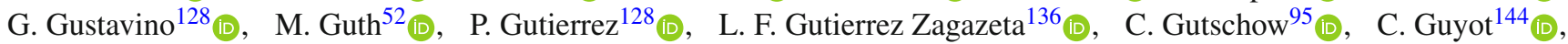

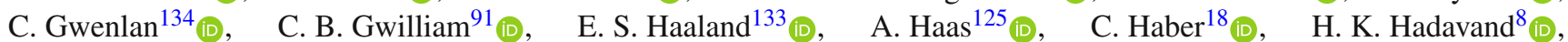
A. Hadef ${ }^{100}$ (D) M. Haleem ${ }^{177}$ (D), J. Haley ${ }^{129}$ (D) J. J. Hall ${ }^{149}$ (D) , G. Halladjian ${ }^{107}$ (D), G. D. Hallewell ${ }^{102}$ (D), K. Hamano ${ }^{176}$ (D),

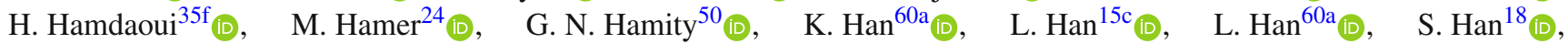

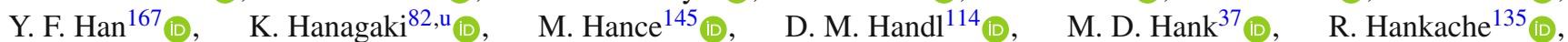

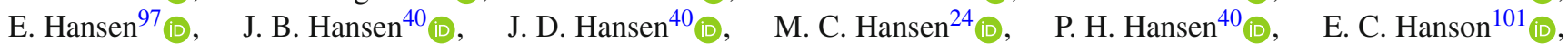
K. $\operatorname{Hara}^{169}$ (D), T. Harenberg ${ }^{182}$ (D), S. Harkusha ${ }^{108}$ (D), P. F. Harrison ${ }^{178}$ ， N. M. $\operatorname{Hartman}^{153}$ (D), N. M. $\operatorname{Hartmann}^{114}$ (D),

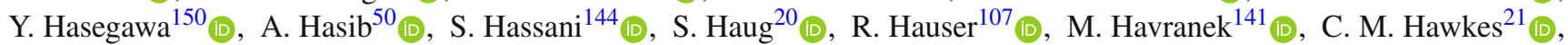

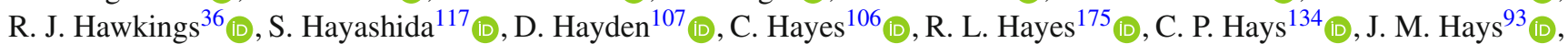

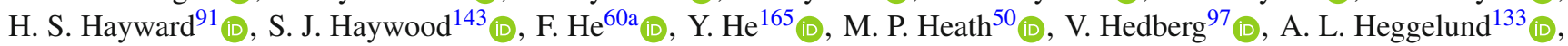
N. D. Hehir ${ }^{93}$ (D) C. Heidegger ${ }^{52}$ (D) K. K. Heidegger ${ }^{52}$ (D), W. D. Heidorn ${ }^{79}$ (D) J. $\operatorname{Heilman}^{34}$ (D), S. Heim ${ }^{46}$ (D) T. Heim ${ }^{18}$ (D),

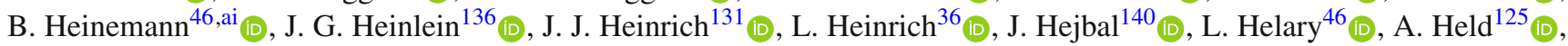
S. Hellesund ${ }^{133}$ (D), C. M. Helling ${ }^{145}$ (D), S. Hellman ${ }^{45 a, 45 b}$ (D), C. Helsens ${ }^{36}$ (D), R.C. W. Henderson ${ }^{90}$, L. Henkelmann ${ }^{32}$ (D),

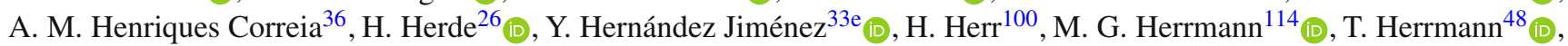

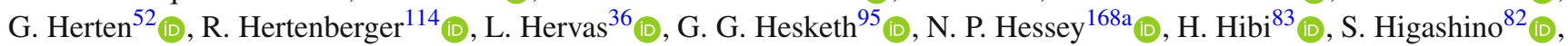
E. Higón-Rodriguez ${ }^{174}$ (D) K. Hildebrand ${ }^{37}$, J. C. Hill ${ }^{32}$ (D) K. K. Hill ${ }^{29}$ (D) K. H. Hiller ${ }^{46}$, S. J. Hillier ${ }^{21}$ (D), M. Hils ${ }^{48}$ (D), I. Hinchliffe ${ }^{18}$ (D), F. Hinterkeuse ${ }^{24}$ (D), M. Hirose ${ }^{132}$ (D), S. Hirose ${ }^{169}$ (D), D. Hirschbuehl ${ }^{182}$ (D), B. Hiti ${ }^{92}$ (D), O. Hladik ${ }^{140}$,

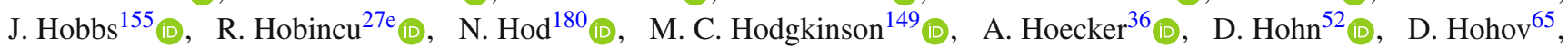
T. Holm ${ }^{24}$ (D), T. R. Holmes ${ }^{37}$ (D), M. Holzbock ${ }^{115}$ (D), L.B.A. H. Hommels ${ }^{32}$ (D), T. M. Hong ${ }^{138}$ (D), J. C. Honig ${ }^{52}$ (D), A. Hönle ${ }^{115}$ (D), B. H. Hooberman ${ }^{173}$ (D), W. H. Hopkins ${ }^{6}$ (D) Y. Horii ${ }^{117}$ (D), P. Horn ${ }^{48}$ (D) L. A. Horyn ${ }^{37}$ (D), S. Hou ${ }^{158}$ (D), A. Hoummada ${ }^{35 a}$, J. Howarth ${ }^{57}$ (D) J. Hoya ${ }^{89}$ (D), M. Hrabovsky ${ }^{130}$ (D), J. Hrivnac ${ }^{65}$, A. Hrynevich ${ }^{109}$ (D), T. Hryn'ova ${ }^{5}$ (D),

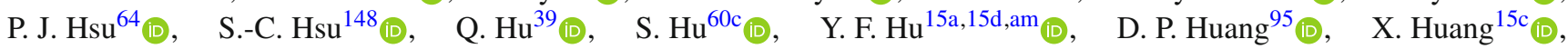

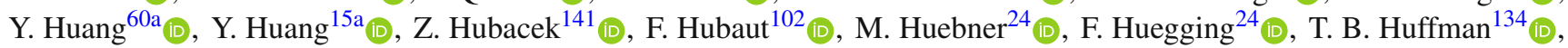

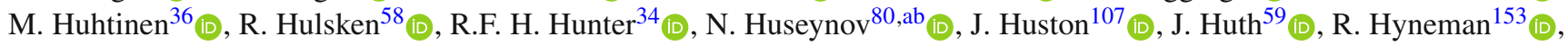

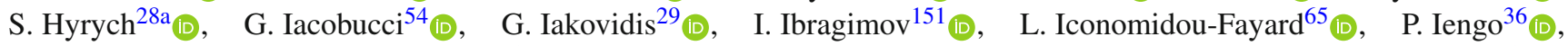
R. Ignazzi ${ }^{40}$, R. Iguchi ${ }^{163}$ (D), T. Iizawa ${ }^{54}$ (D), Y. Ikegami ${ }^{82}$ (D), M. Ikeno ${ }^{82}$ (D) N. Ilic ${ }^{119,167, \text { aa }, ~ F . ~ I l t z s c h e ~}{ }^{48}$, H. Imam $^{35 a}$ (D),

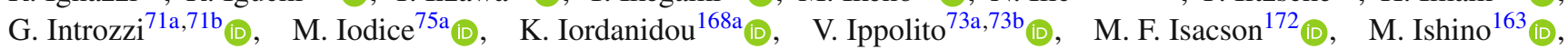
W. Islam ${ }^{129}$ (D) C. Issever ${ }^{19,46}$ (D), S. Istin ${ }^{160}$ (D) J. M. Iturbe Ponce ${ }^{63 a}$ (D) R. Iuppa ${ }^{76 a, 76 b}$ (D), A. Ivina ${ }^{180}$ (D), J. M. Izen ${ }^{43}$ (D),

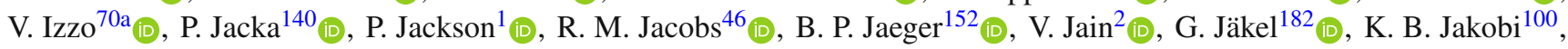

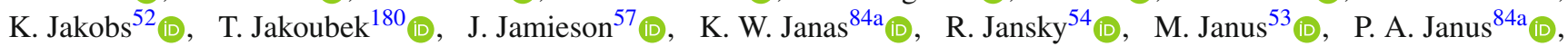
G. Jarlskog ${ }^{97}$ (D), A. E. Jaspan ${ }^{91}$ (i), N. Javadov ${ }^{80, a b}$, T. Javưrek ${ }^{36}$ (D) M. Javurkova ${ }^{103}$ (D), F. Jeanneau ${ }^{144}$ (i), L. Jeanty ${ }^{131}$ (i),

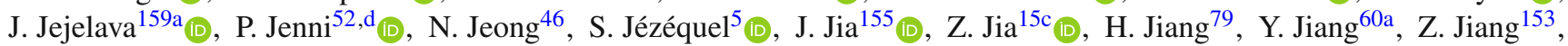

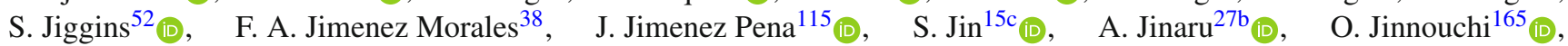

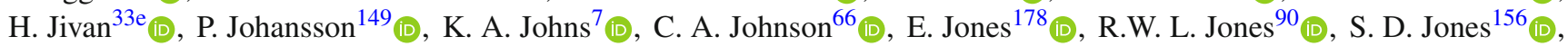

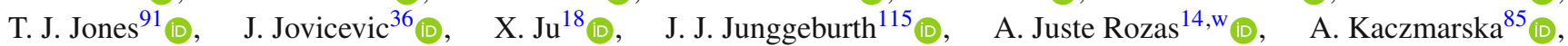
M. Kado ${ }^{73 a}, 73 b$, H. Kagan ${ }^{127}$ (D), M. Kagan ${ }^{153}$ (D), A. Kahn ${ }^{39}$, C. Kahra ${ }^{100}$ (D), T. Kaji ${ }^{179}$ (D), E. Kajomovitz ${ }^{160}$ (D), $\begin{array}{lll}\text { C. W. Kalderon } & 29 & \text { (D) }, \quad \text { A. Kaluza }\end{array}$

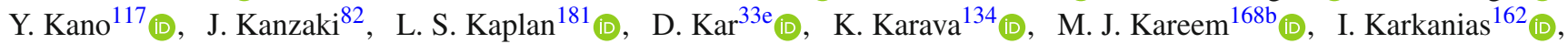

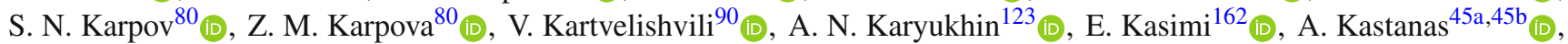

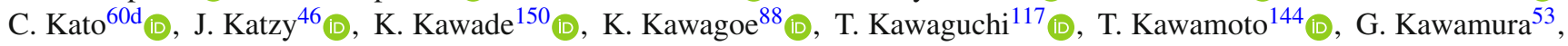

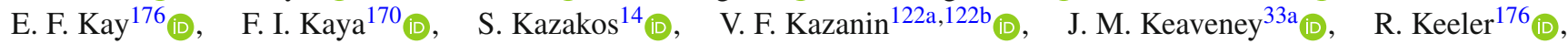

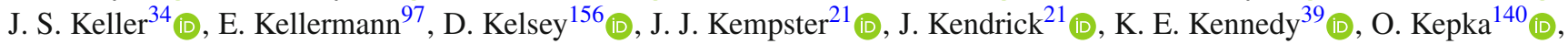
S. Kersten ${ }^{182}$ (D), B. P. Kerševan ${ }^{92}$ (D), S. Ketabchi Haghighat ${ }^{167}$ (D), F. Khalil-Zada ${ }^{13}$, M. Khandoga ${ }^{144}$ (D), A. Khanov ${ }^{129}$ (D),

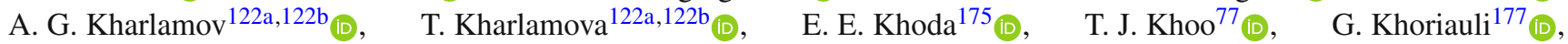
E. Khramov ${ }^{80}$ (D), J. Khubua ${ }^{159 b}$ (D), S. Kido ${ }^{83}$ (D), M. Kiehn ${ }^{36}$ (D), E. $\operatorname{Kim}^{165}$ (D), Y. K. Kim ${ }^{37}$ (D), N. Kimura ${ }^{95}$ (D), A. Kirchhoff ${ }^{53}$ (D) D. $\operatorname{Kirchmeier}^{48}$ (D) J. Kirk $^{143}$ (D) A. E. Kiryunin ${ }^{115}$ (D), T. Kishimoto ${ }^{163}$ (D), D. P. Kisliuk ${ }^{167}$, V. Kitali ${ }^{46}$ (D), C. Kitsaki ${ }^{10}$ (D), O. Kivernyk ${ }^{24}$ (D), T. Klapdor-Kleingrothaus ${ }^{52}$ (D), M. Klassen ${ }^{61 \mathrm{a}}$ (D)， C. Klein ${ }^{34}$ (D), M. H. Klein ${ }^{106}$ (D),

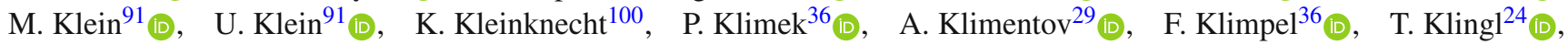

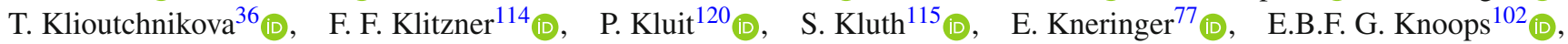




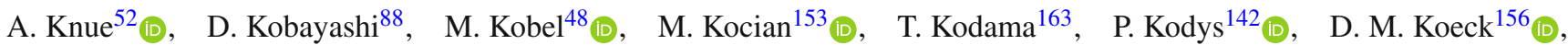

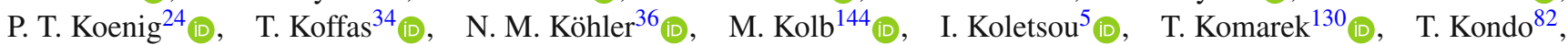
K. Köneke $e^{52}$ (D), A.X. Y. Kong ${ }^{1}$ (D), A. C. König ${ }^{119}$ (D), T. Kono ${ }^{126}$ (D), V. Konstantinides ${ }^{95}, \quad$ N. Konstantinidis ${ }^{95}$ (D), B. Konya ${ }^{97}$ (D), R. Kopeliansky ${ }^{66}$ (D), S. Koperny ${ }^{84 a} a_{(D)}, \quad$ K. Korcyl ${ }^{85}$ (D), K. Kordas ${ }^{162}$ (D), G. Koren ${ }^{161}$, A. Korn ${ }^{95}$ (D), I. Korolkov ${ }^{14}$ (D) E. V. Korolkova ${ }^{149}$ ， N. Korotkova ${ }^{113}$ (D), O. Kortner ${ }^{115}$ (D), S. Kortner ${ }^{115}$ (D), V. V. Kostyukhin ${ }^{149,166}$ (D), A. Kotsokechagia ${ }^{65}$ (D) A. Kotwal $^{49}$ (D), A. Koulouris ${ }^{10}$ (D), A. Kourkoumeli-Charalampidi ${ }^{71 a, 71 b}$ (D), C. Kourkoumelis ${ }^{9}$ (D), E. Kourlitis ${ }^{6}$ (D) V. Kouskoura ${ }^{29}$ (D), R. Kowalewski ${ }^{176}$ (D) W. Kozanecki ${ }^{101}$ (D), A. S. Kozhin ${ }^{123}$ (D), V. A. Kramarenko ${ }^{113}$ (D),

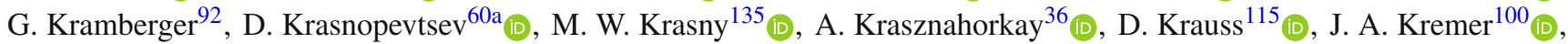

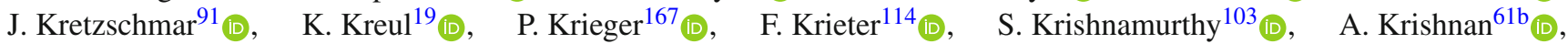

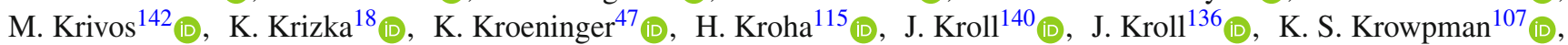

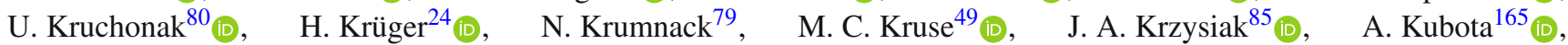
O. Kuchinskaia ${ }^{16} 6_{(D)}$, S. Kuday ${ }^{4 b}$ (D) , D. Kuechler ${ }^{46}$ (D), J. T. Kuechler ${ }^{46}$ (D), S. Kuehn ${ }^{36}$ (D), T. Kuhl ${ }^{46}$ (D), V. Kukhtin ${ }^{80}$ (D), Y. Kulchitsky ${ }^{108, a e}$ (D), S. Kuleshov ${ }^{146 b}{ }_{(\mathbb{D})}$, Y. P. Kulinich ${ }^{173}$, M. Kuna ${ }^{58}$ (D), A. Kupco ${ }^{140}$ (D), T. Kupfer ${ }^{47}$, O. Kuprash ${ }^{52}$ (D), H. Kurashige ${ }^{83}$ (D) L. L. Kurchaninov ${ }^{168 a}$ (D), Y. A. Kurochkin ${ }^{108}$ (D) A. Kurova ${ }^{112}$ (D), M. G. Kurth ${ }^{15 a}, 15 d$, E. S. Kuwertz ${ }^{36}$ (D), M. Kuze ${ }^{165}$ (D), A. K. Kvam ${ }^{148}$ (D), J. Kvita ${ }^{130}$ (D), T. Kwan ${ }^{104}$ (D), C. Lacasta ${ }^{174}$ (D), F. Lacava ${ }^{73 a, 73 b}$ (D), D.P. J. Lack ${ }^{101}$ (D),

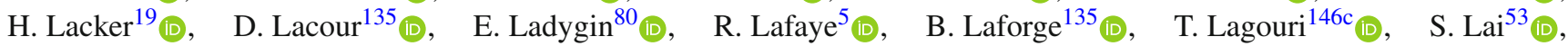

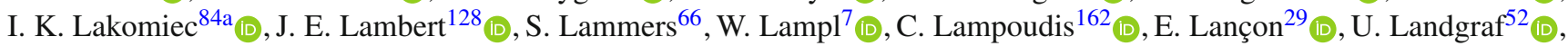
M.P. J. Landon ${ }^{93}$ (D), V. S. Lang ${ }^{52}$ (i), J. C. Lange $e^{53}$ (i), R. J. Langenberg ${ }^{103}$ (I), A. J. Lankford ${ }^{171}$ (i), F. Lanni ${ }^{29}$ (D),

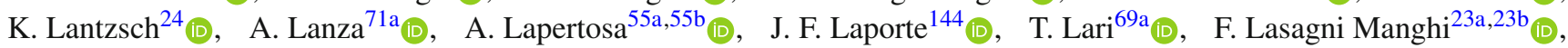

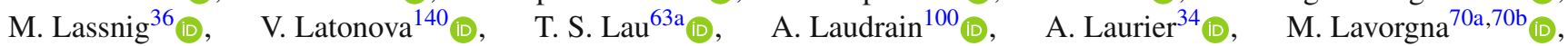

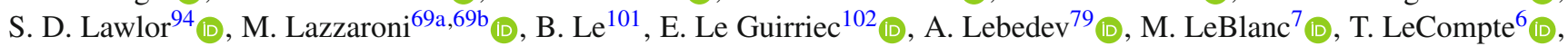

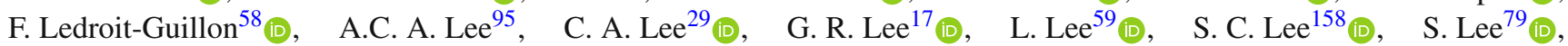

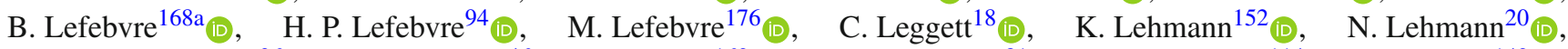

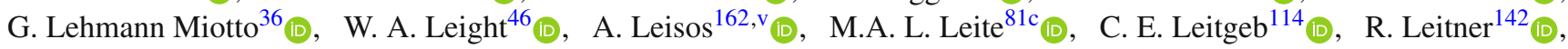
K. J. C. Leney ${ }^{42}$ (D), T. Lenz ${ }^{24}$ (D), S. Leone ${ }^{72 a}$ (D), C. Leonidopoulos ${ }^{50}$ (D), A. Leopold ${ }^{135}$ (D) C. Leroy ${ }^{110}$ (D) R. Les ${ }^{107}$ (D),

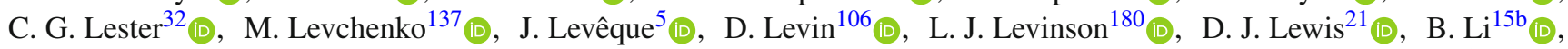

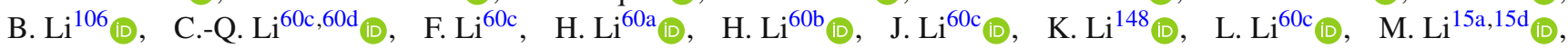

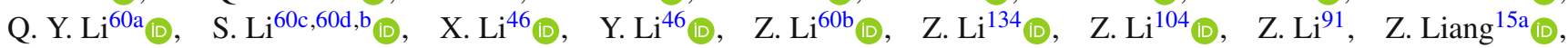

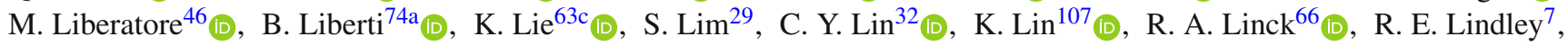

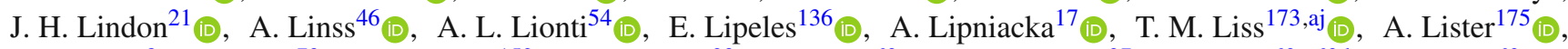

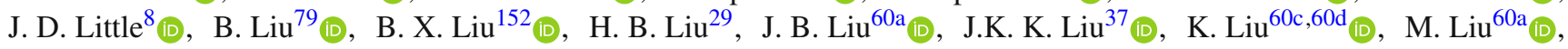

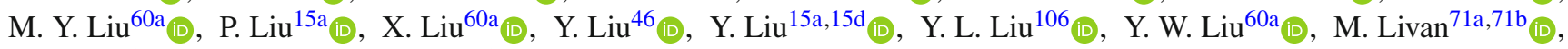

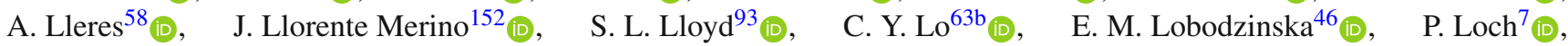

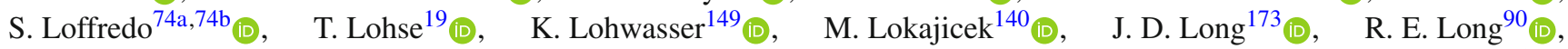
I. Longarini ${ }^{73 a}, 73 b$ (D), L. Longo ${ }^{36}$, I. Lopez Paz ${ }^{101}$, A. Lopez Solis ${ }^{149}$ (D), J. Lorenz ${ }^{114}$ (D), N. Lorenzo Martinez ${ }^{5}$ (1),

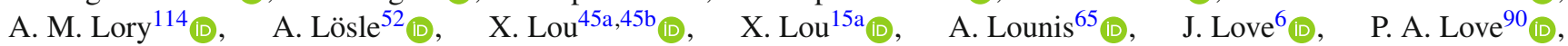

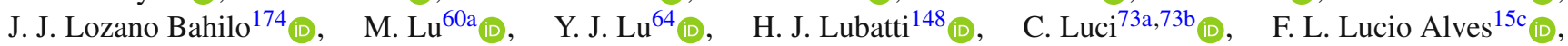

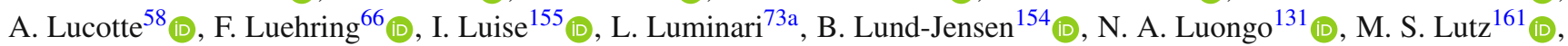

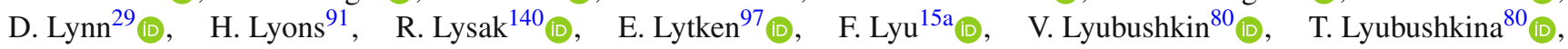

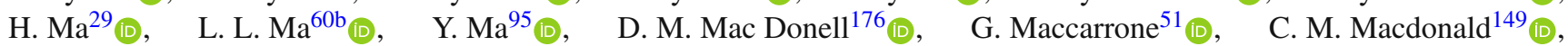

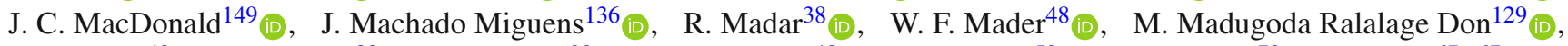

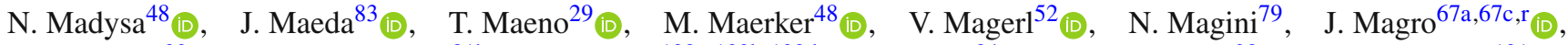

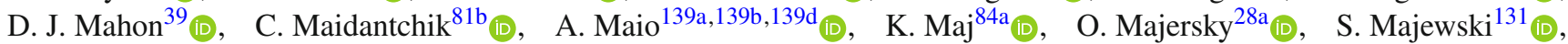
Y. Makida ${ }^{82}$, N. Makovec ${ }^{65}$ (), B. Malaescu ${ }^{135}$ (D), Pa. Malecki ${ }^{85}$ (D), V. P. Maleev ${ }^{137}$ (D), F. Malek ${ }^{58}$ (D), D. Malito ${ }^{41 \mathrm{a}, 41 \mathrm{~b}}$ (D), U. Mallik ${ }^{78}$ (D) C. Malone ${ }^{32}$ (I), S. Maltezos ${ }^{10}$, S. Malyukov ${ }^{80}$, J. Mamuzic ${ }^{174}$ (D), G. Mancini ${ }^{51}$ (D), J. P. Mandalia ${ }^{93}$ (D), I. Mandić92 (D), L. Manhaes de Andrade Filho ${ }^{81 a}$ (D), I. M. Maniatis ${ }^{162}$ (D) J. Manjarres $\operatorname{Ramos}^{48}$ (D), K. H. Mankinen ${ }^{97}$ (D), A. Mann ${ }^{114}$ (D), A. Manousos ${ }^{77}$ (D), $\quad$ B. Mansoulie ${ }^{144}$ (D), I. Manthos ${ }^{162}$ (1), S. Manzoni ${ }^{120}$ (D), A. Marantis ${ }^{162}$ (D),

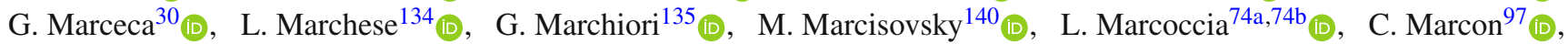
M. Marjanovic ${ }^{128}$ (D) Z. Marshall ${ }^{18}$ (D), M.U. F. Martensson ${ }^{172}$ (D), S. Marti-Garcia ${ }^{174}$ (D), C. B. Martin ${ }^{127}$ (D), T. A. Martin ${ }^{178}$ (D), V. J. Martin ${ }^{50}$ (D) $\quad$ B. Martin dit Latour ${ }^{17}$ (D), L. Martinelli ${ }^{75 a, 75 b}$ (D) $\quad$ M. Martinez ${ }^{14, w}$ (D),$\quad$ P. Martinez Agullo ${ }^{174}$ (D), V. I. Martinez Outschoorn ${ }^{103}$ (D), S. Martin-Haugh ${ }^{143}$ (D), V. S. Martoiu ${ }^{27 b}$ (D), A. C. Martyniuk ${ }^{95}$ (D), A. Marzin ${ }^{36}$ (D),

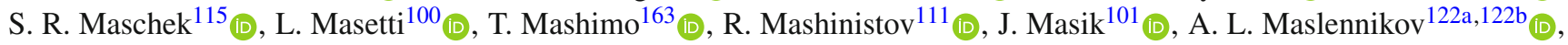

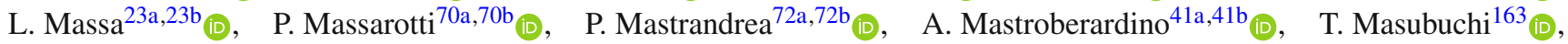




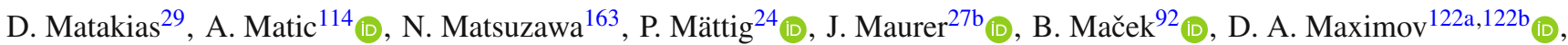

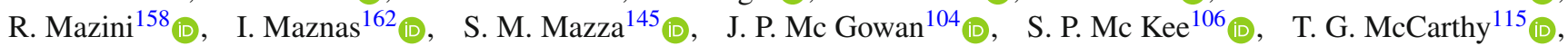

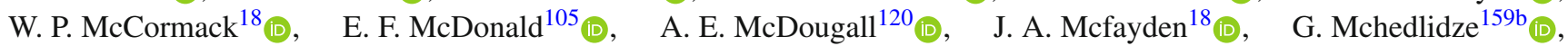

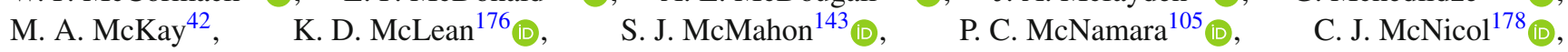

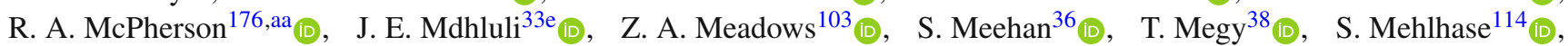

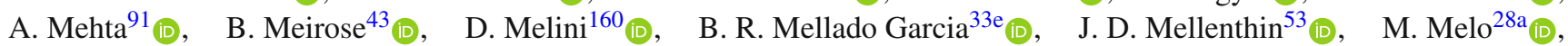
F. Meloni ${ }^{46}(\mathbb{D})$ A. Melzer $^{24}$ (D), E. D. Mendes Gouveia ${ }^{139 a, 139 e}{ }_{(\mathbb{D})}$, A. M. Mendes Jacques Da Costa ${ }^{21}$ (D), H. Y. Meng ${ }^{167}$, L. Meng ${ }^{36}$ (D), X. T. Meng ${ }^{106}(\mathbb{D}), \quad$ S. Menke ${ }^{115}\left(\mathbb{D}, \quad\right.$ E. Meoni ${ }^{41 \mathrm{a}, 41 \mathrm{~b}} \mathbb{( D D}_{\mathrm{D}}, \quad$ S. Mergelmeyer ${ }^{19}, \quad$ S.A. M. Merkt ${ }^{138}$,

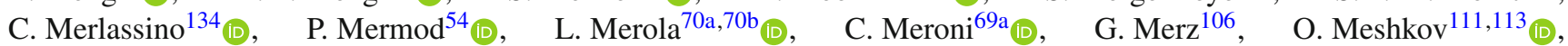
J.K. R. Meshreki ${ }^{151}\left(\mathbb{D}, \quad\right.$ J. Metcalfe ${ }^{6}(\mathbb{D}), \quad$ A. S. Mete ${ }^{6}$ (D) $\quad$ C. Meyer ${ }^{66}(\mathbb{D}), \quad$ J.-P. Meyer ${ }^{144}\left(\mathbb{D}, \quad\right.$ M. Michetti ${ }^{19}{ }_{(\mathbb{D})}$, R. P. Middleton ${ }^{143}$ (D), L. Mijović ${ }^{50}$ (D), G. Mikenberg ${ }^{180}$ (D), M. Mikestikova ${ }^{140}$ (D), M. Mikuž $\check{z}^{92}$ (D), H. Mildner ${ }^{149}$ (D),

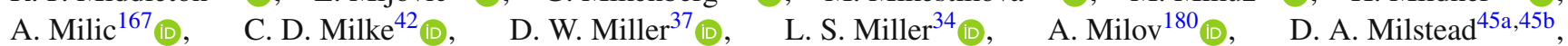

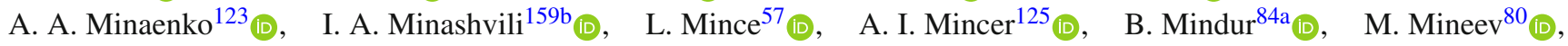
Y. Minegishi ${ }^{163}$, Y. Mino ${ }^{86}$ (D), L. M. Mir $^{14}$ (D), M. Mironova ${ }^{134}$, T. Mitani ${ }^{179}$ (D), J. Mitrevski ${ }^{114}$, V. A. Mitsou ${ }^{174}$ (D),

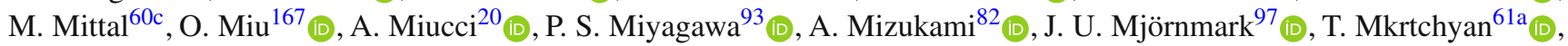

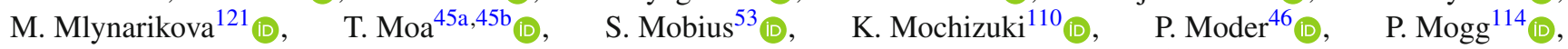
S. Mohapatra ${ }^{39}$ (D) R. Moles-Valls ${ }^{24}$ (D) K. Mönig ${ }^{46}$ (D), E. Monnier ${ }^{102}$ (D), A. Montalbano ${ }^{152}$ (D), J. Montejo Berlingen ${ }^{36}$ (D),

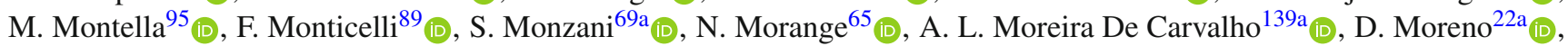
M. Moreno Llácer ${ }^{174}$ (D), C. Moreno Martinez ${ }^{14}$ (D), P. Morettini ${ }^{55 b}{ }_{(\mathbb{C})}, \quad$ M. Morgenstern ${ }^{160}$ (D), S. Morgenstern ${ }^{48}$ (D), D. Mori ${ }^{152}$ (D) M. Morii ${ }^{59}$ (D) M. Morinaga ${ }^{179}$ (D), V. Morisbak ${ }^{133}$ (D) A. K. Morley ${ }^{36}$ (D) , G. Mornacchi ${ }^{36}$ (D) , A. P. Morris ${ }^{95}$ (D),

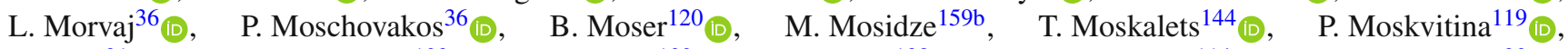
J. Moss ${ }^{31, n}$ (D), E. J. W. Moyse ${ }^{103}$ (D), S. Muanza ${ }^{102}$ (D), J. Mueller ${ }^{138}$ (D), R.S. P. Mueller ${ }^{114}$, D. Muenstermann ${ }^{90} \mathbb{C}_{\text {(D) }}$,

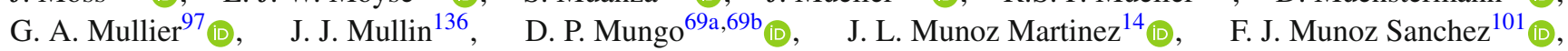
P. Murin ${ }^{28 b}$ (D), W. J. Murray ${ }^{143,178}$ (D), A. Murrone ${ }^{69 a, 69 b}$ (D), J. M. Muse ${ }^{128}$ (D), M. Muškinja ${ }^{18}$ (D), C. Mwewa ${ }^{33 a}$ (D),

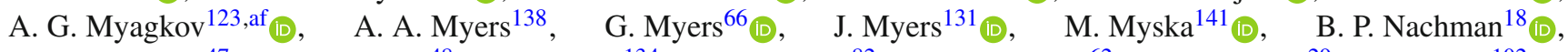
O. Nackenhorst ${ }^{47}$ (D), A.Nag Nag ${ }^{48}$ (D), K. Nagai ${ }^{134}$ (D), K. Nagano ${ }^{82}$ (D), Y. Nagasaka ${ }^{62}$ (D), J. L. Nagle ${ }^{29}$ (D), E. Nagy ${ }^{102}$ (D),

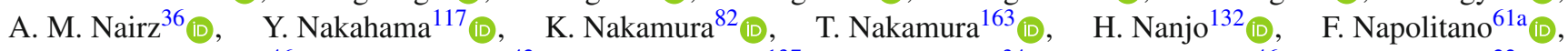

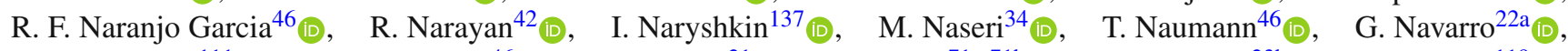

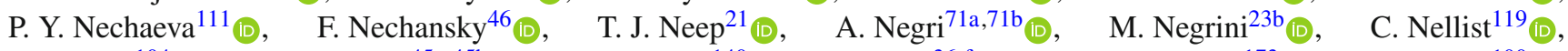

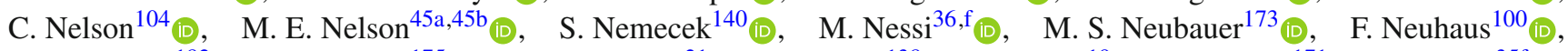

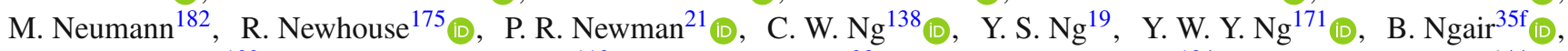

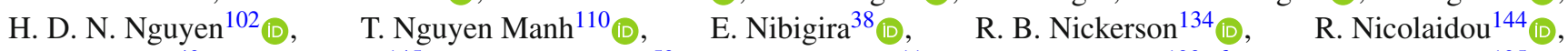

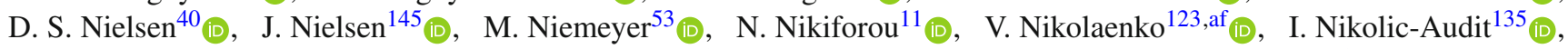
K. Nikolopoulos ${ }^{21}$ (D), P. Nilsson ${ }^{29}$ (D) H. R. Nindhito ${ }^{54}$ (D), A. Nisati ${ }^{73 \mathrm{a}}$ (i), N. Nishu ${ }^{60 \mathrm{c}}$ (D) R. Nisius ${ }^{115}$ (D), I. Nitsche ${ }^{47}$, T. Nitta ${ }^{179}$ (D), T. Nobe ${ }^{163}$ (D), D. L. Noel $^{32}$ (D), Y. Noguchi ${ }^{86}$ (D), I. Nomidis ${ }^{135}$ (D), M. A. Nomura ${ }^{29}$, M. Nordberg ${ }^{36}$,

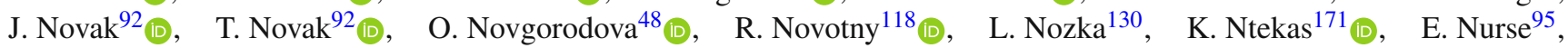

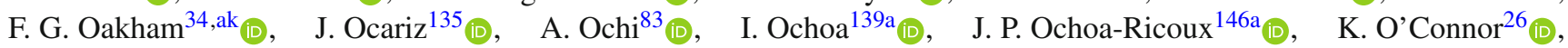

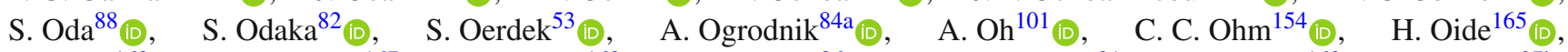
R. Oishi ${ }^{163}$ (D), M. L. Ojeda ${ }^{167}$ (D) H. Okawa ${ }^{169}$ (D), Y. Okazaki ${ }^{86}$ (D) M. W. O'Keefe ${ }^{91}$, Y. Okumura ${ }^{163}$ (D), A. Olariu ${ }^{27 b}$,

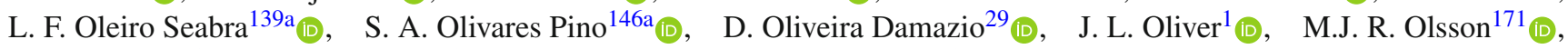
A. Olszewski ${ }^{85}$ (D), J. Olszowska ${ }^{85}$ (D), Ö. O. Öncel ${ }^{24}$ (D), D. C. O’Neil ${ }^{152}$ (D), A. P. O’neill ${ }^{134}$ (D), A. Onofre ${ }^{139 a, 139 e}$ (D), P.U. E. Onyisi ${ }^{11}$ (D) H. Oppen ${ }^{133}$, R. G. Oreamuno Madriz ${ }^{121}$, M. J. Oreglia ${ }^{37} \mathbb{D}$, G. E. Orellana ${ }^{89}$ (D) D. Orestano ${ }^{75 a}, 75 b$ (D),

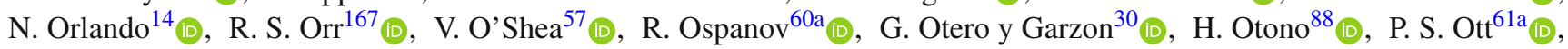

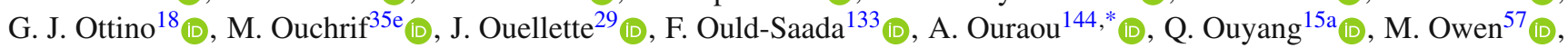

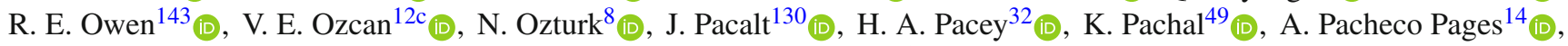

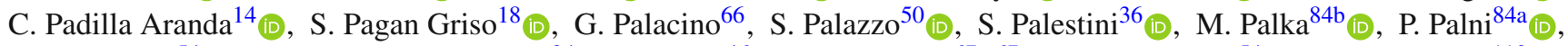

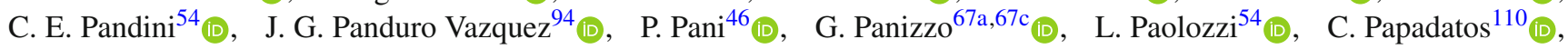

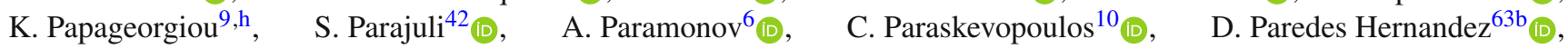
S. R. Paredes Saenz ${ }^{134}$ (I),$\quad$ B. Parida ${ }^{180}$ (D), T. H. Park ${ }^{167}$ (D), A. J. Parker ${ }^{31}$ (D), M. A. Parker ${ }^{32}$ (D), F. Parodi ${ }^{55 a, 55 b}$ (D), E. W. Parrish ${ }^{121}$ (D) J. A. Parsons ${ }^{39}$ (D) U. Parzefall ${ }^{52}$ (D) L. Pascual Dominguez ${ }^{135}$ (D) , V. R. Pascuzzi ${ }^{18}$ (D) J.M. P. Pasner ${ }^{145}$ (D),

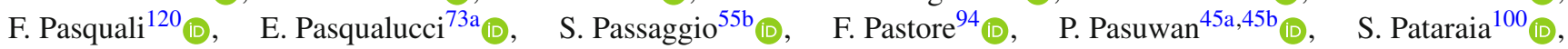

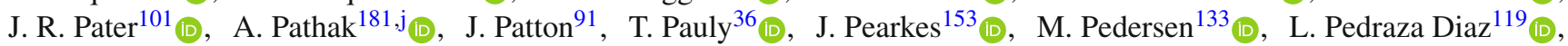

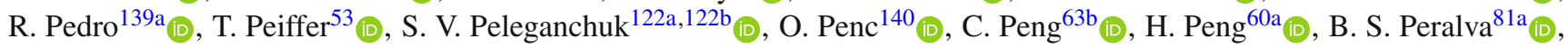




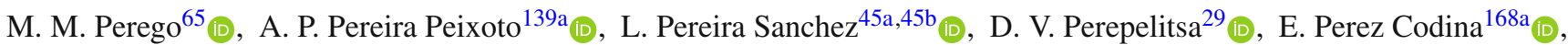

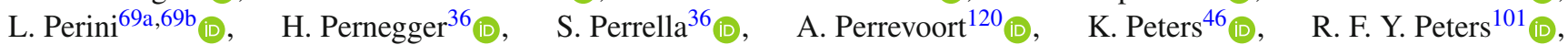

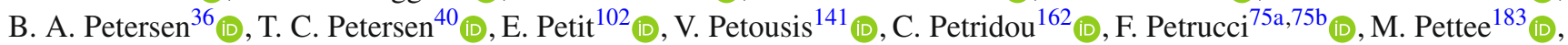

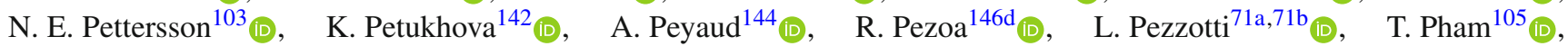

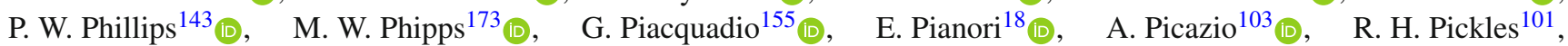

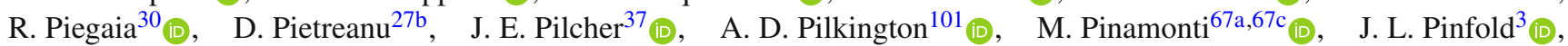
C. Pitman Donaldson ${ }^{95}$, M. Pitt ${ }^{161}$ (D), L. Pizzimento ${ }^{74 a, 74 b}{ }_{(D)}, \quad$ A. Pizzini ${ }^{120}{ }_{(\mathbb{D})}, \quad$ M.-A. Pleier ${ }^{29}$ (D), V. Plesanovs ${ }^{52}$, V. Pleskot ${ }^{142}$ (D), E. Plotnikova ${ }^{80}, \quad$ P. Podberezko ${ }^{122 a, 122 b}$ (D) $_{1}, \quad$ R. Poettgen ${ }^{97}$ (D), R. Poggi ${ }^{54}$ (D), L. Poggioli $^{135}$ (D),

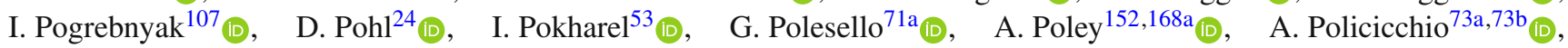
R. Polifka ${ }^{142}$ (D), A. Polini ${ }^{23 b}{ }_{(D)}$, C. S. Pollard ${ }^{46}$, V. Polychronakos ${ }^{29}$ (D), D. Ponomarenko ${ }^{112}$ (D), L. Pontecorvo ${ }^{36}$ (D),

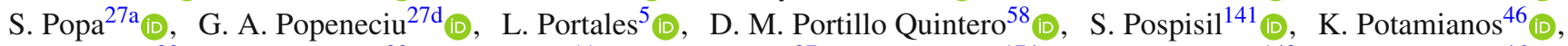

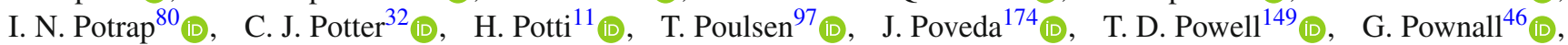
M. E. Pozo Astigarraga ${ }^{36}$ (D) A. Prades Ibanez ${ }^{174}$ (D), P. Pralavorio ${ }^{102}$ (D), M. M. Prapa ${ }^{44}$ (D) S. Prell ${ }^{79}$ (D), D. Price ${ }^{101}$ (D),

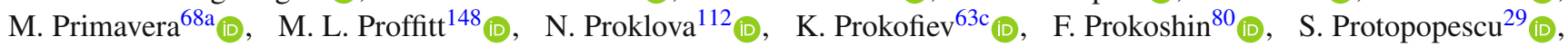

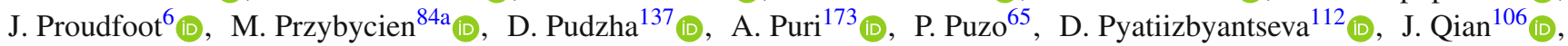
Y. Qin ${ }^{101}$ (D), A. Quadt ${ }^{53}$ (D), M. Queitsch-Maitland ${ }^{36}$ (D), G. Rabanal Bolanos ${ }^{59}$ (D), M. Racko ${ }^{28 a}$, F. Ragusa ${ }^{69 a}, 69 b$ (D),

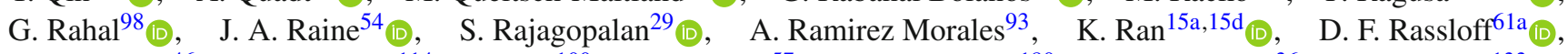

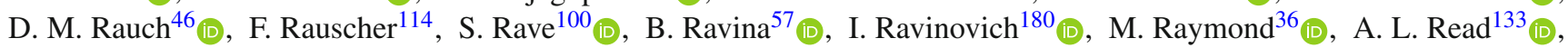

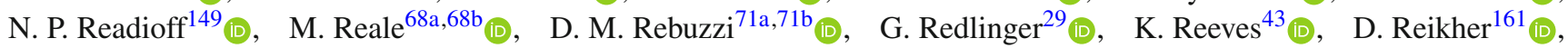

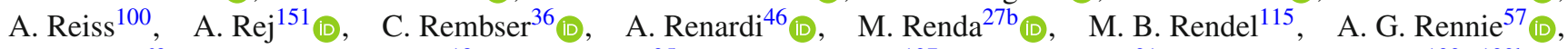

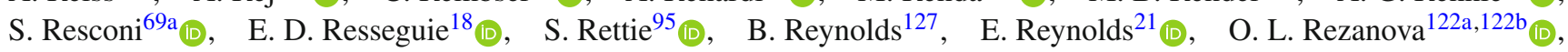

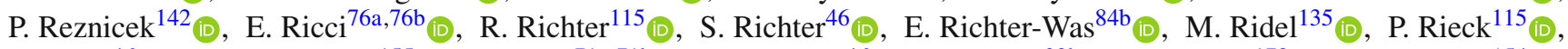

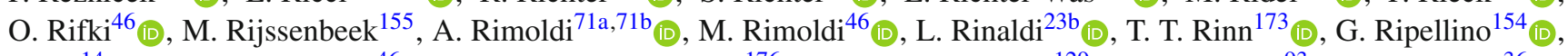

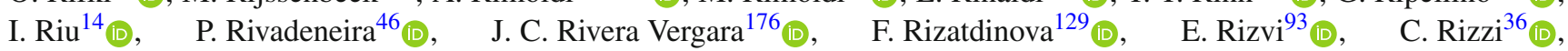

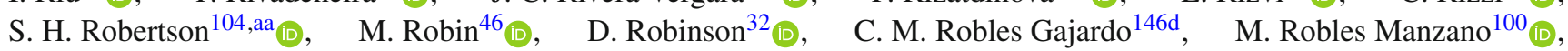

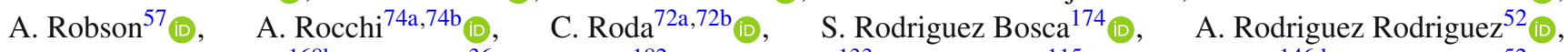

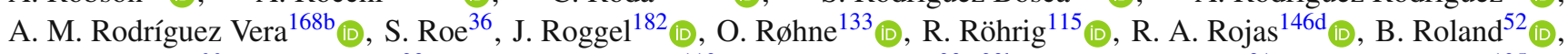
C.P. A. Roland ${ }^{66}$ (D), J. Roloff ${ }^{29}$ (D), A. Romaniouk ${ }^{112}$ (D), M. Romano ${ }^{23 a, 23 b}{ }_{(\mathbb{C})}, \quad$ N. Rompotis ${ }^{91}$ (D), M. Ronzani ${ }^{125}$ (D),

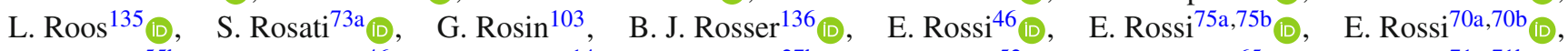

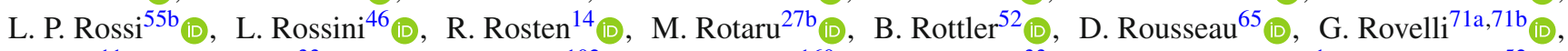

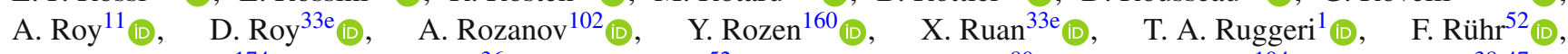
A. Ruiz-Martinez ${ }^{174}$ (D), A. Rummler ${ }^{36}$ (D) Z. Rurikova ${ }^{52}$ (D), N. A. Rusakovich ${ }^{80}$ (D) H. L. Russel1 ${ }^{104}$ (I) , L. Rustige ${ }^{38,47}$ (D),

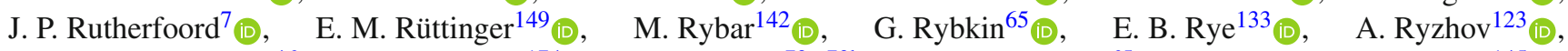

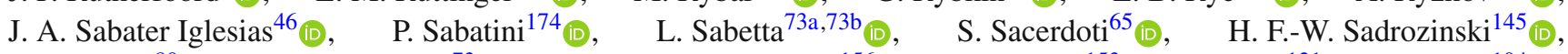

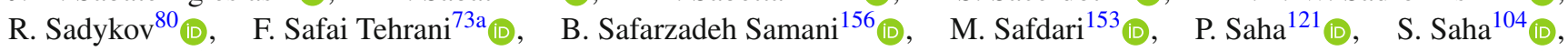

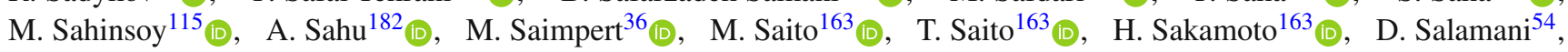

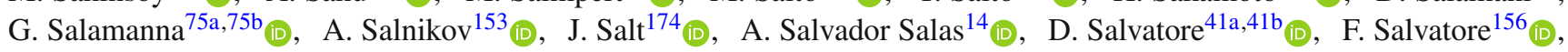

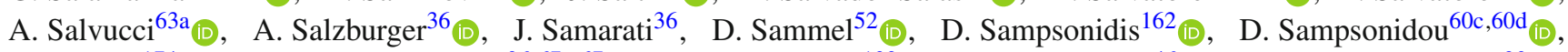

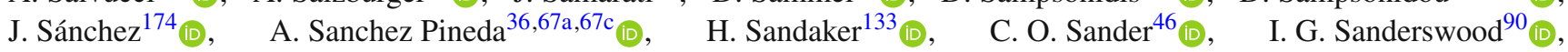

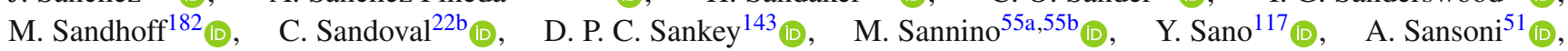

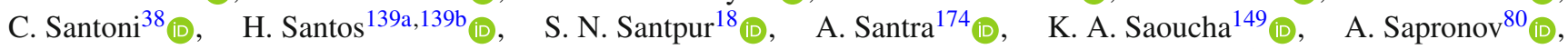

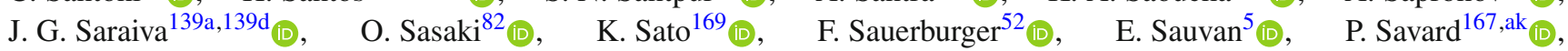
R. Sawada ${ }^{163}$ (D) , C. Sawyer ${ }^{143}$ (D), L. Sawyer ${ }^{96}$ (D) I. Sayago Galvan ${ }^{174}$, C. Sbarra ${ }^{23 b}$ (D), A. Sbrizzi ${ }^{67 a, 67 c}$ (D) T. Scanlon ${ }^{95}$ (D),

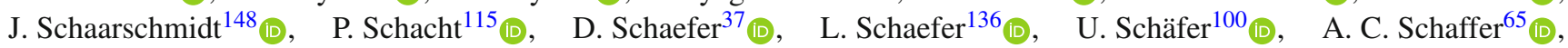

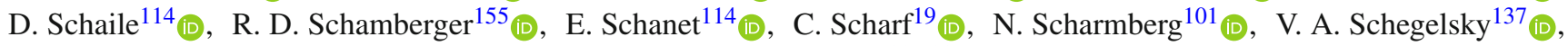

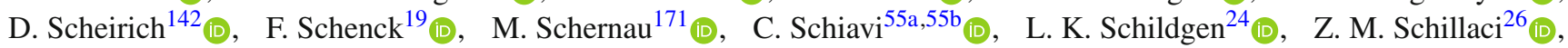

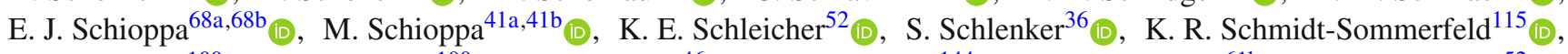

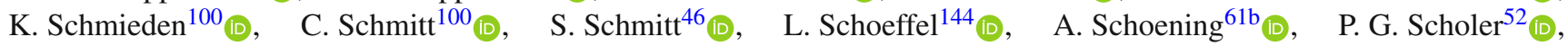
E. Schopf ${ }^{134}$ (D), M. Schott ${ }^{100}$ (D), J.F. P. Schouwenberg ${ }^{119}$ (D), J. Schovancova ${ }^{36}$ (D), S. Schramm ${ }^{54}$ (D), F. Schroeder ${ }^{182}$ (D),

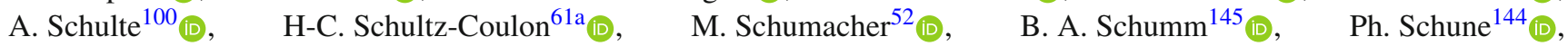
A. Schwartzman ${ }^{153}$ (D) T. A. Schwarz ${ }^{106}$ (D) Ph. Schwemling ${ }^{144}$ (D), R. Schwienhorst ${ }^{107}$ (D), A. Sciandra ${ }^{145}$ (D) , G. Sciolla ${ }^{26}$ (D),

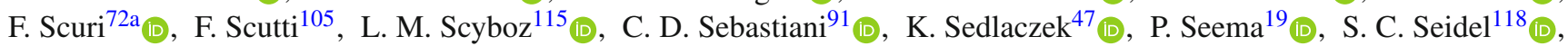

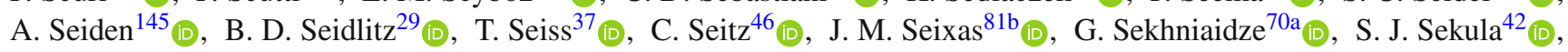




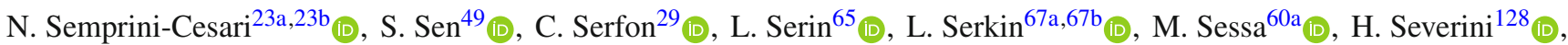
S. Sevova ${ }^{153}$ (D), F. Sforza ${ }^{55 a, 55 b}$ (I), A. Sfyrla ${ }^{54}$ (D), E. Shabalina ${ }^{53}$ (D), J. D. Shahinian ${ }^{136}$ (I) , N. W. Shaikh ${ }^{45 a, 45 b}$ (D),

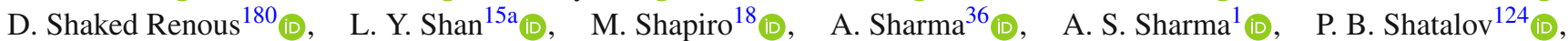

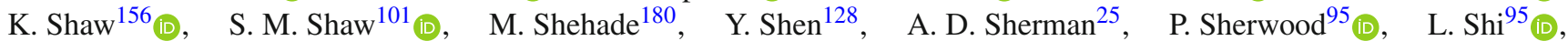
C. O. Shimmin ${ }^{183}$ (D), Y. Shimogama ${ }^{179}$ (D), M. Shimojima ${ }^{116}$ (D), J. D. Shinner ${ }^{94}$ (D), I.P. J. Shipsey ${ }^{134}$ (D), S. Shirabe ${ }^{165}$ (D), $^{\circ}$,

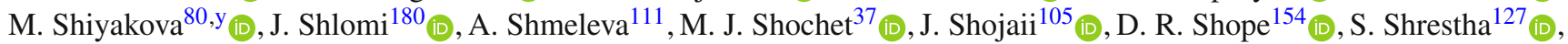

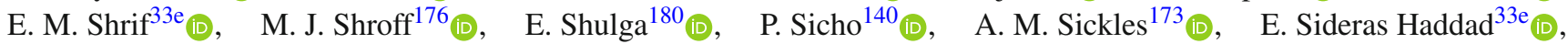

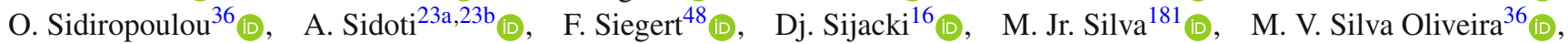

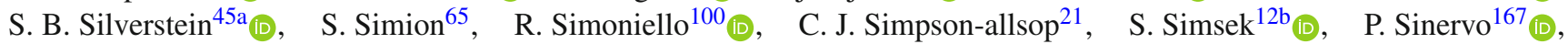

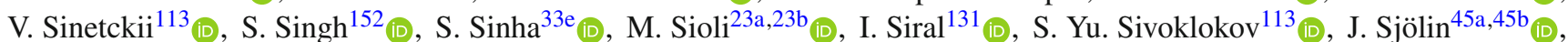

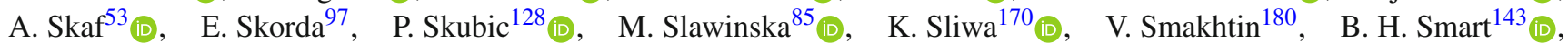

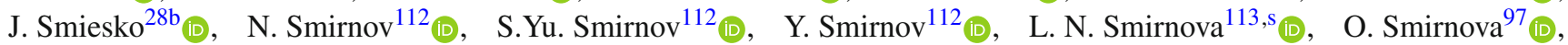

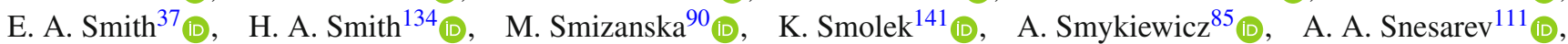

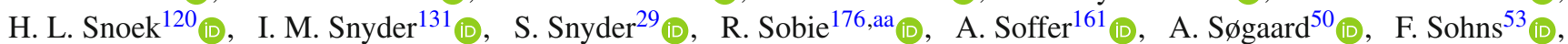

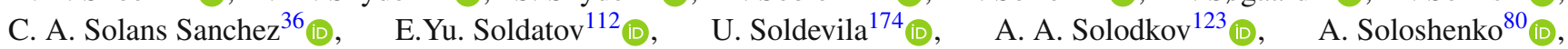

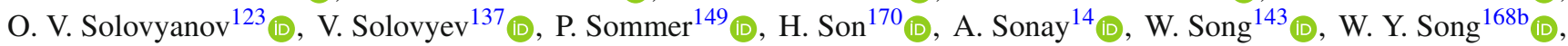

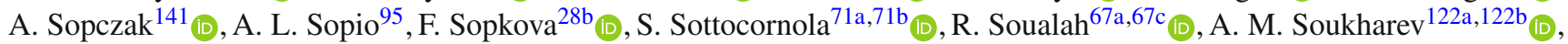

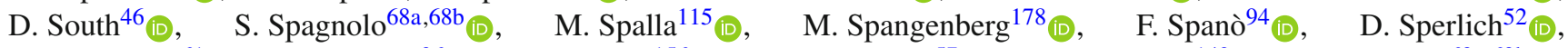

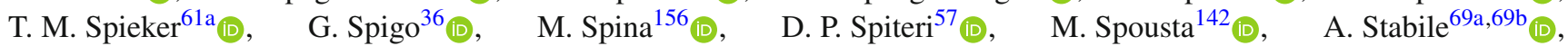

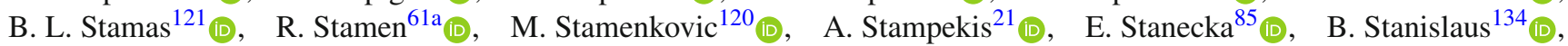

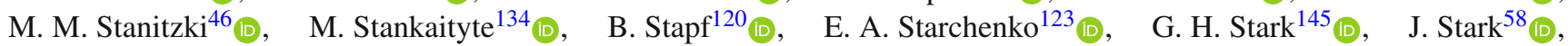

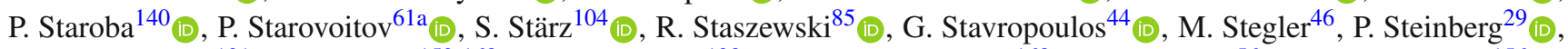

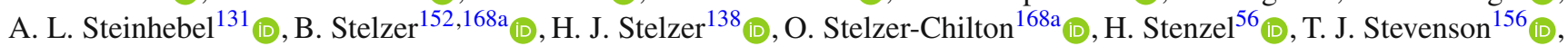

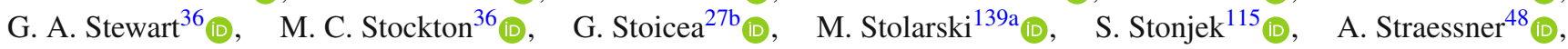
J. Strandberg ${ }^{154}$ (D), S. Strandberg ${ }^{45 a, 45 b}$ (D), M. Strauss ${ }^{128}$ (D), T. Strebler ${ }^{102}$ (I),$\quad$ P. Strizenec ${ }^{28 b}$ (D), R. Ströhmer ${ }^{177}$ (D),

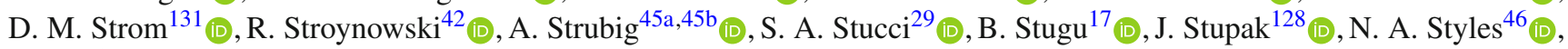

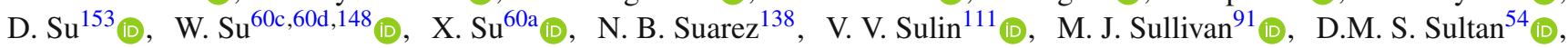

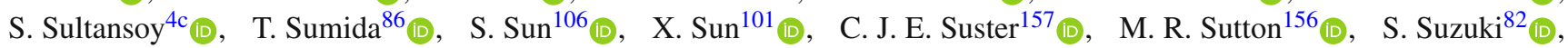

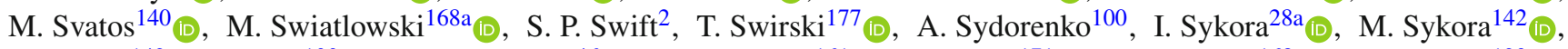
T. Sykora ${ }^{142}$ (D), D. Ta ${ }^{100}$ (D), K. Tackmann ${ }^{46, x}$ (D), J. Taenzer ${ }^{161}, \quad$ A. Taffard ${ }^{171}$ (D), R. Tafirout ${ }^{168 a}$ (ID), E. Tagiev ${ }^{123}$ (D), R. H. M. Taibah ${ }^{135}$ (D), R. Takashima ${ }^{87}$, K. Takeda ${ }^{83}$ (D) T. Takeshita ${ }^{150}$ (D), E. P. Takeva ${ }^{50}$ (D), Y. Takubo ${ }^{82}$ (D) M. Talby ${ }^{102}$ (D), A. A. Talyshev ${ }^{122 a, 122 b}$ (D), K. C. Tam ${ }^{63 b}, \quad$ N. M. Tamir ${ }^{161}, \quad$ J. Tanaka ${ }^{163}$ (D), R. Tanaka ${ }^{65}$ (D), S. Tapia Araya ${ }^{173}$ (D),

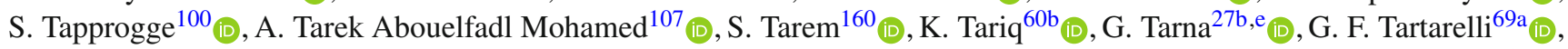

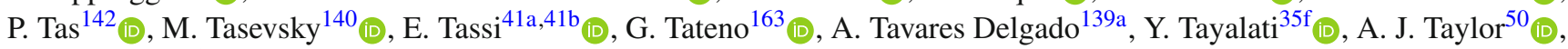

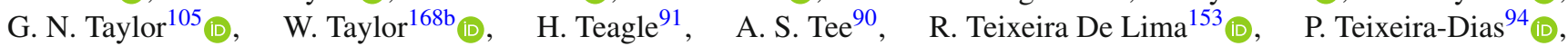

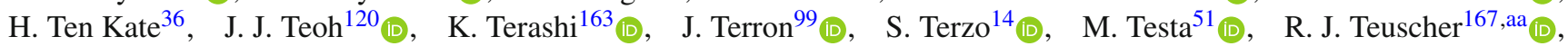

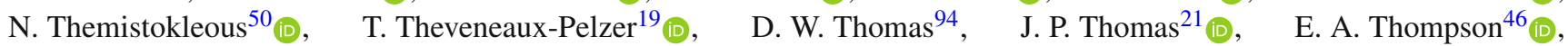

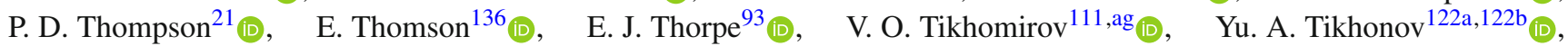

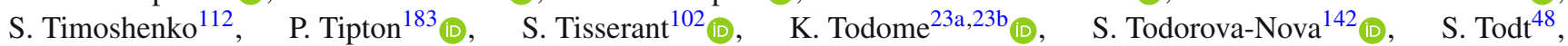

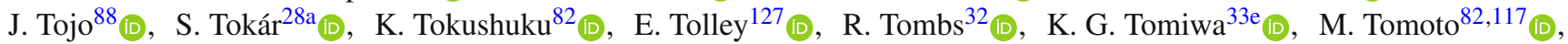

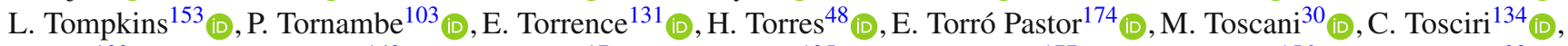
J. Toth $^{102, z}$ (D), D. R. Tovey ${ }^{149}$ (D), A. Traeet ${ }^{17}$, C. J. Treado ${ }^{125}$ (D), T. Trefzger ${ }^{177}$ (D), F. Tresoldi ${ }^{156}$ (D), A. Tricoli ${ }^{29}$ (D),

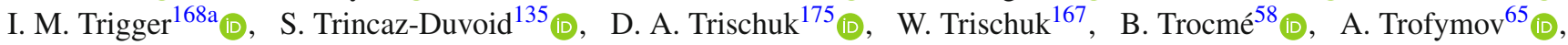

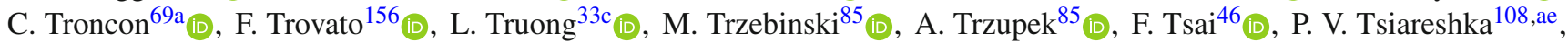
A. Tsirigotis ${ }^{162, \mathrm{v}}$ (D) , V. Tsiskaridze ${ }^{155}$ (D), E. G. Tskhadadze ${ }^{159 a}$, M. Tsopoulou ${ }^{162}$ (D) I. I. Tsukerman ${ }^{124}$ (D), V. Tsulaia ${ }^{18}$ (D),

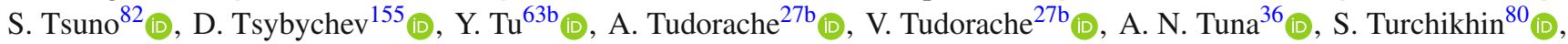

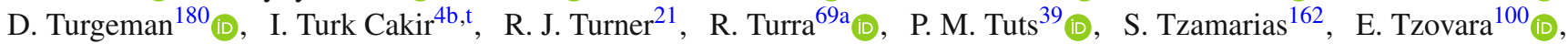

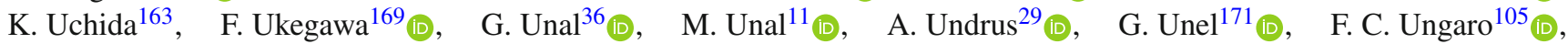

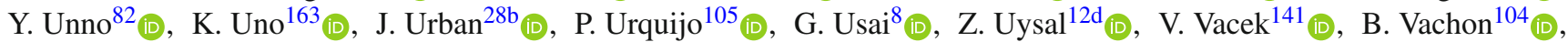

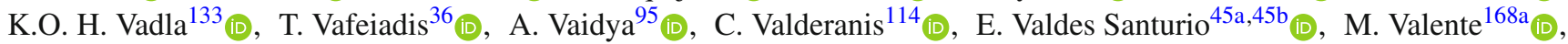
S. Valentinetti ${ }^{23 a, 23 b}{ }_{(1)}, \quad$ A. Valero ${ }^{174}\left(\mathbb{D}, \quad\right.$ L. Valéry ${ }^{46}$ (D) $\quad$ R. A. Vallance ${ }^{21}$ (D), A. Vallier ${ }^{36}$ (D), J. A. Valls Ferrer ${ }^{174}$,

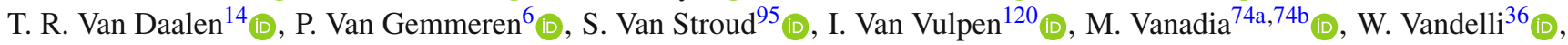

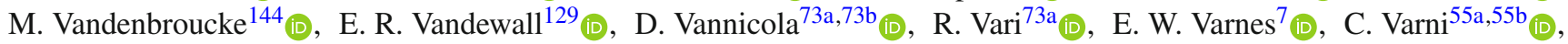




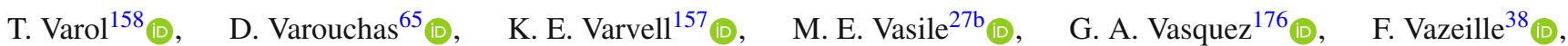
D. Vazquez Furelos ${ }^{14}$ (D), T. Vazquez Schroeder ${ }^{36}$ (D) J. Veatch ${ }^{53}$ (D), V. Vecchio ${ }^{101}$ (D), M. J. Veen ${ }^{120}$ (D), L. M. Veloce ${ }^{167}$ (D),

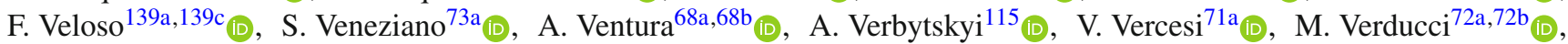
C. M. Vergel Infante ${ }^{79}$, C. Vergis ${ }^{24}$ (D), W. Verkerke ${ }^{120}$ (D), A. T. Vermeulen ${ }^{120}$ (D), J. C. Vermeulen ${ }^{120}$ (D), C. Vernieri ${ }^{153}$ (D),

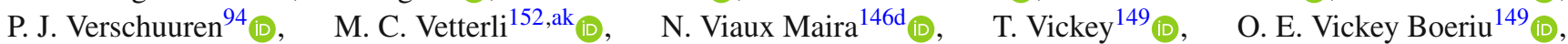

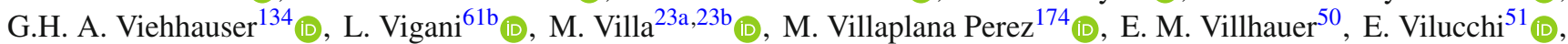

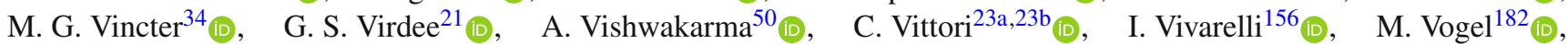

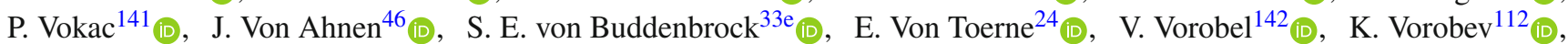

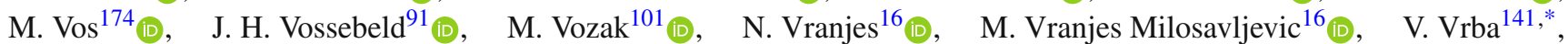
M. Vreeswijk ${ }^{120}$ (D), N. K. Vu ${ }^{102}$ (D), R. Vuillermet ${ }^{36}$ (D), I. Vukotic ${ }^{37}$ (D), S. Wada ${ }^{169}$ (D), P. Wagner ${ }^{24}$ (D), W. Wagner ${ }^{182}$ (D), J. Wagner-Kuhr ${ }^{114}$ (D), S. Wahdan ${ }^{182}$ (D), H. Wahlberg ${ }^{89}$ (D), R. Wakasa ${ }^{169}$ (D), V. M. Walbrecht ${ }^{115}$ (D), J. Walder ${ }^{143}$ (D), R. Walker ${ }^{14}$ (I),$\quad$ S. D. Walker ${ }^{94}$, W. Walkowiak ${ }^{151}$ (I), V. Wallangen ${ }^{45 a, 45 b}, \quad$ A. M. Wang ${ }^{59}$ (I), A. Z. Wang ${ }^{181}$ (D),

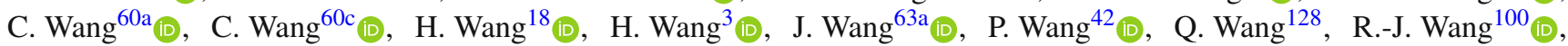

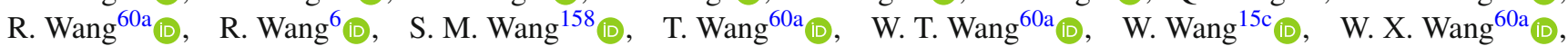

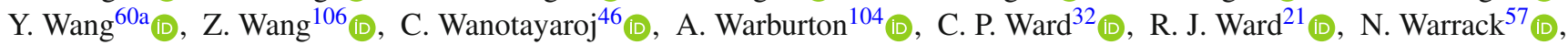
A. T. Watson ${ }^{21}$ (D) M. F. Watson ${ }^{21}$ (D), G. Watts ${ }^{148}$ (D), B. M. Waugh ${ }^{95}$ (D) A. F. Webb ${ }^{11}$ (D) C. Weber ${ }^{29}$ (D) M. S. Weber ${ }^{20}$ (D), S. A. Weber ${ }^{34}$ (D) S. M. Weber ${ }^{61 \mathrm{a}}$ (D), Y. Wei ${ }^{134}$ (D) A. R. Weidberg ${ }^{134}$ (D) , J. Weingarten ${ }^{47}$ (D) M. Weirich ${ }^{100}$ (D), C. Weiser ${ }^{52}$ (D),

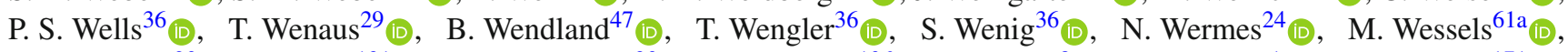

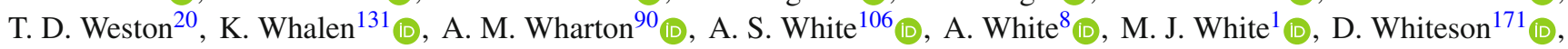

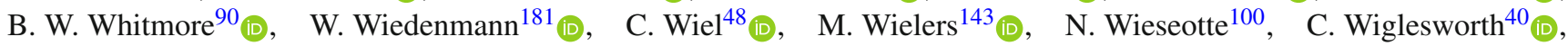
L.A. M. Wiik-Fuchs ${ }^{52}$ (D) H. G. Wilkens ${ }^{36}$ (D) L. J. Wilkins ${ }^{94}$ (i) D. M. Williams ${ }^{39}$ (1), H. H. Williams ${ }^{136}$, S. Williams ${ }^{32}$ (D), S. Willocq ${ }^{103}$ (D) P. J. Windischhofer ${ }^{134}$ (D), I. Wingerter-Seez ${ }^{5}$ (D) E. Winkels ${ }^{156}$ (D), F. Winklmeier ${ }^{131}$ (D), B. T. Winter ${ }^{52}$ (D),

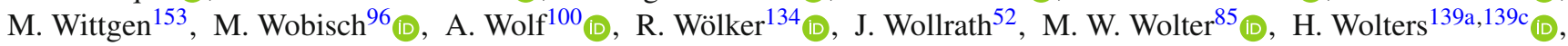

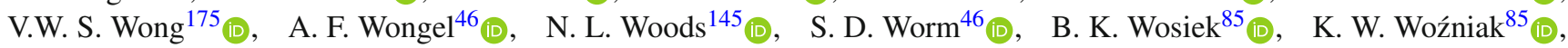

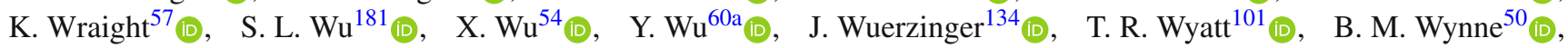

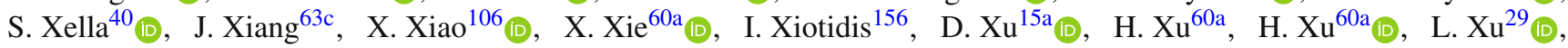

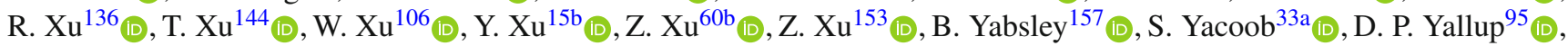
N. Yamaguchi ${ }^{88}$ (D), Y. Yamaguchi ${ }^{165}$ (D), A. Yamamoto ${ }^{82}$ (D), M. Yamatani ${ }^{163}, \quad$ T. Yamazaki ${ }^{163}$ (D), Y. Yamazaki ${ }^{83}$ (D),

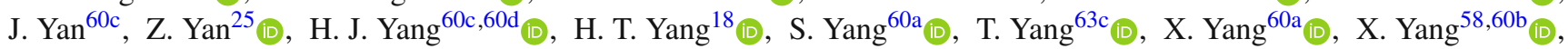

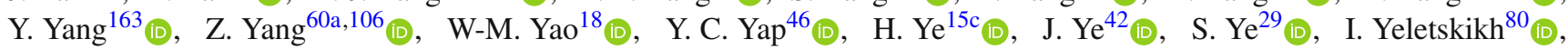
M. R. Yexley ${ }^{90}$ (D), E. Yigitbasi ${ }^{25}$ (D), P. Yin ${ }^{39}$ (D), K. Yorita ${ }^{179}$ (D), K. Yoshihara ${ }^{79}$ (D), C.J. S. Young ${ }^{36}$ (D), C. Young ${ }^{153}$ (D),

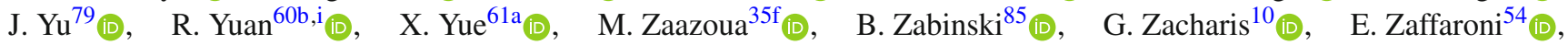

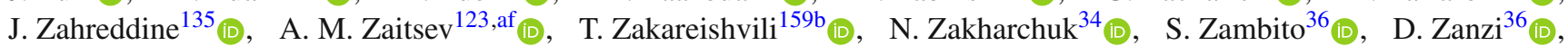

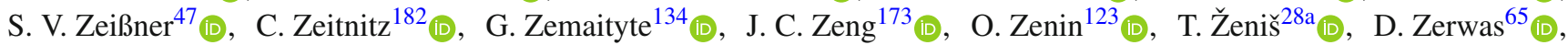

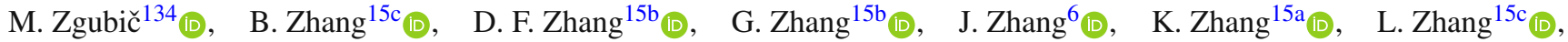

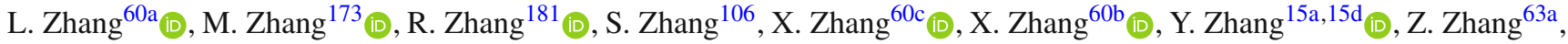

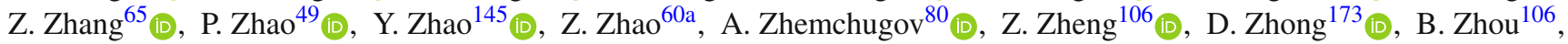

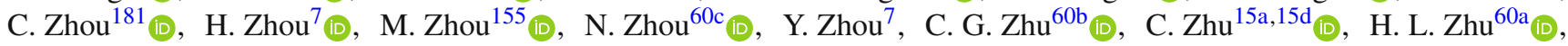

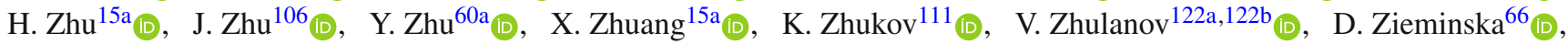

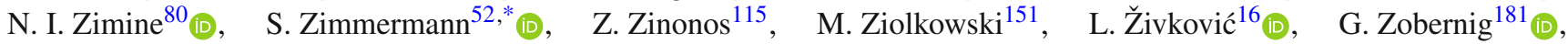
A. Zoccoli ${ }^{23 a}, 23 b$ (D) K. Zoch ${ }^{53}$ (D) , T. G. Zorbas ${ }^{149}$ (D), R. Zou ${ }^{37}$ (D) L. Zwalinski ${ }^{36}$ (D)

\footnotetext{
${ }^{1}$ Department of Physics, University of Adelaide, Adelaide, Australia

${ }^{2}$ Physics Department, SUNY Albany, Albany, NY, USA

${ }^{3}$ Department of Physics, University of Alberta, Edmonton, AB, Canada

4 (a) Department of Physics, Ankara University, Ankara, Turkey; ${ }^{(b)}$ Istanbul Aydin University, Application and Research

Center for Advanced Studies, Istanbul, Turkey; ${ }^{(c)}$ Division of Physics, TOBB University of Economics and Technology,

Ankara, Turkey

${ }^{5}$ LAPP, Université Grenoble Alpes, Université Savoie Mont Blanc, CNRS/IN2P3, Annecy, France

${ }^{6}$ High Energy Physics Division, Argonne National Laboratory, Argonne, IL, USA

${ }^{7}$ Department of Physics, University of Arizona, Tucson, AZ, USA

${ }^{8}$ Department of Physics, University of Texas at Arlington, Arlington, TX, USA

${ }^{9}$ Physics Department, National and Kapodistrian University of Athens, Athens, Greece
} 
${ }^{10}$ Physics Department, National Technical University of Athens, Zografou, Greece

${ }^{11}$ Department of Physics, University of Texas at Austin, Austin, TX, USA

12 (a) Bahcesehir University, Faculty of Engineering and Natural Sciences, Istanbul, Turkey; ${ }^{(b)}$ Istanbul Bilgi University, Faculty of Engineering and Natural Sciences, Istanbul, Turkey; ${ }^{\left({ }^{c}\right)}$ Department of Physics, Bogazici University, Istanbul, Turkey; ${ }^{(\mathrm{d})}$ Department of Physics Engineering, Gaziantep University, Gaziantep, Turkey

${ }^{13}$ Institute of Physics, Azerbaijan Academy of Sciences, Baku, Azerbaijan

${ }^{14}$ Institut de Física d'Altes Energies (IFAE), Barcelona Institute of Science and Technology, Barcelona, Spain

15 (a) Institute of High Energy Physics, Chinese Academy of Sciences, Beijing, China; ${ }^{(b)}$ Physics Department, Tsinghua University, Beijing, China; ${ }^{(c)}$ Department of Physics, Nanjing University, Nanjing, China; ${ }^{(d)}$ University of Chinese Academy of Science (UCAS), Beijing, China

${ }^{16}$ Institute of Physics, University of Belgrade, Belgrade, Serbia

${ }^{17}$ Department for Physics and Technology, University of Bergen, Bergen, Norway

${ }^{18}$ Physics Division, Lawrence Berkeley National Laboratory and University of California, Berkeley, CA, USA

${ }^{19}$ Institut für Physik, Humboldt Universität zu Berlin, Berlin, Germany

${ }^{20}$ Albert Einstein Center for Fundamental Physics and Laboratory for High Energy Physics, University of Bern, Bern, Switzerland

${ }^{21}$ School of Physics and Astronomy, University of Birmingham, Birmingham, UK

22 (a) Facultad de Ciencias y Centro de Investigaciónes, Universidad Antonio Nariño, Bogotá, Colombia; ${ }^{(b)}$ Departamento de Física, Universidad Nacional de Colombia, Bogotá, Colombia

23 (a) Dipartimento di Fisica, INFN Bologna and Universita' di Bologna, Bologna, Italy; ${ }^{(b)}$ INFN Sezione di Bologna, Bologna, Italy

${ }^{24}$ Physikalisches Institut, Universität Bonn, Bonn, Germany

${ }^{25}$ Department of Physics, Boston University, Boston, MA, USA

${ }^{26}$ Department of Physics, Brandeis University, Waltham, MA, USA

27 (a) Transilvania University of Brasov, Brasov, Romania; ${ }^{(b)}$ Horia Hulubei National Institute of Physics and Nuclear Engineering, Bucharest, Romania; ${ }^{(c)}$ Department of Physics, Alexandru Ioan Cuza University of Iasi, Iasi, Romania; ${ }^{(d)}$ National Institute for Research and Development of Isotopic and Molecular Technologies, Physics Department, Cluj-Napoca, Romania; ${ }^{(\mathrm{e})}$ University Politehnica Bucharest, Bucharest, Romania; ${ }^{(\mathrm{f})}$ West University in Timisoara, Timisoara, Romania

28 (a) Faculty of Mathematics, Physics and Informatics, Comenius University, Bratislava, Slovak Republic; ${ }^{(b)}$ Department of Subnuclear Physics, Institute of Experimental Physics of the Slovak Academy of Sciences, Kosice, Slovak Republic

${ }^{29}$ Physics Department, Brookhaven National Laboratory, Upton, NY, USA

${ }^{30}$ Departamento de Física, Universidad de Buenos Aires, Buenos Aires, Argentina

${ }^{31}$ California State University, Long Beach, CA, USA

${ }^{32}$ Cavendish Laboratory, University of Cambridge, Cambridge, UK

33 (a) Department of Physics, University of Cape Town, Cape Town, South Africa; ${ }^{(b)}$ iThemba Labs, Western Cape, South Africa; ${ }^{(c)}$ Department of Mechanical Engineering Science, University of Johannesburg, Johannesburg, South Africa; (d) University of South Africa, Department of Physics, Pretoria, South Africa; ${ }^{(e)}$ School of Physics, University of the Witwatersrand, Johannesburg, South Africa

${ }^{34}$ Department of Physics, Carleton University, Ottawa, ON, Canada

35 (a) Faculté des Sciences Ain Chock, Réseau Universitaire de Physique des Hautes Energies - Université Hassan II, Casablanca, Morocco; ${ }^{(b)}$ Faculté des Sciences, Université Ibn-Tofail, Kénitra, Morocco; ${ }^{(c)}$ Faculté des Sciences Semlalia, Université Cadi Ayyad, LPHEA-Marrakech, Morocco; ${ }^{(d)}$ Moroccan Foundation for Advanced Science Innovation and Research (MAScIR), Rabat, Morocco; ${ }^{(e)}$ LPMR, Faculté des Sciences, Université Mohamed Premier, Oujda, Morocco; ${ }^{(\mathrm{f})}$ Faculté des sciences, Université Mohammed V, Rabat, Morocco

${ }^{36}$ CERN, Geneva, Switzerland

${ }^{37}$ Enrico Fermi Institute, University of Chicago, Chicago, IL, USA

${ }^{38}$ LPC, Université Clermont Auvergne, CNRS/IN2P3, Clermont-Ferrand, France

${ }^{39}$ Nevis Laboratory, Columbia University, Irvington, NY, USA

${ }^{40}$ Niels Bohr Institute, University of Copenhagen, Copenhagen, Denmark

41 (a) Dipartimento di Fisica, Università della Calabria, Rende, Italy; ${ }^{(b)}$ Laboratori Nazionali di Frascati, INFN Gruppo Collegato di Cosenza, Frascati, Italy

${ }^{42}$ Physics Department, Southern Methodist University, Dallas, TX, USA 
43 Physics Department, University of Texas at Dallas, Richardson, TX, USA

${ }^{44}$ National Centre for Scientific Research "Demokritos", Agia Paraskevi, Greece

45 (a) Department of Physics, Stockholm University, Stockholm, Sweden; ${ }^{(b)}$ Oskar Klein Centre, Stockholm, Sweden

46 Deutsches Elektronen-Synchrotron DESY, Hamburg and Zeuthen, Germany

${ }^{47}$ Lehrstuhl für Experimentelle Physik IV, Technische Universität Dortmund, Dortmund, Germany

${ }^{48}$ Institut für Kern- und Teilchenphysik, Technische Universität Dresden, Dresden, Germany

49 Department of Physics, Duke University, Durham, NC, USA

${ }^{50}$ SUPA-School of Physics and Astronomy, University of Edinburgh, Edinburgh, UK

51 INFN e Laboratori Nazionali di Frascati, Frascati, Italy

52 Physikalisches Institut, Albert-Ludwigs-Universität Freiburg, Freiburg, Germany

53 II. Physikalisches Institut, Georg-August-Universität Göttingen, Göttingen, Germany

54 Département de Physique Nucléaire et Corpusculaire, Université de Genève, Geneva, Switzerland

55 (a) Dipartimento di Fisica, Università di Genova, Genoa, Italy; (b) INFN Sezione di Genova, Genoa, Italy

56 II. Physikalisches Institut, Justus-Liebig-Universität Giessen, Giessen, Germany

57 SUPA-School of Physics and Astronomy, University of Glasgow, Glasgow, UK

58 LPSC, Université Grenoble Alpes, CNRS/IN2P3, Grenoble INP, Grenoble, France

${ }^{59}$ Laboratory for Particle Physics and Cosmology, Harvard University, Cambridge, MA, USA

60 (a) State Key Laboratory of Particle Detection and Electronics, Department of Modern Physics, University of Science and Technology of China, Hefei, China; ${ }^{(b)}$ Key Laboratory of Particle Physics and Particle Irradiation (MOE), Institute of Frontier and Interdisciplinary Science, Shandong University, Qingdao, China; (c) School of Physics and Astronomy, Shanghai Jiao Tong University, Key Laboratory for Particle Astrophysics and Cosmology (MOE), SKLPPC, Shanghai, China; ${ }^{(d)}$ Tsung-Dao Lee Institute, Shanghai, China

61 (a) Kirchhoff-Institut für Physik, Ruprecht-Karls-Universität Heidelberg, Heidelberg, Germany; ${ }^{(b)}$ Physikalisches Institut, Ruprecht-Karls-Universität Heidelberg, Heidelberg, Germany

62 Faculty of Applied Information Science, Hiroshima Institute of Technology, Hiroshima, Japan

63 (a) Department of Physics, Chinese University of Hong Kong, Shatin N.T., Hong Kong; ${ }^{(b)}$ Department of Physics, University of Hong Kong, Pok Fu Lam, Hong Kong; ${ }^{(c)}$ Department of Physics and Institute for Advanced Study, Hong Kong University of Science and Technology, Clear Water Bay, Kowloon, Hong Kong, China

${ }^{64}$ Department of Physics, National Tsing Hua University, Hsinchu, Taiwan

65 IJCLab, CNRS/IN2P3, Université Paris-Saclay, 91405 Orsay, France

${ }^{66}$ Department of Physics, Indiana University, Bloomington, IN, USA

67 (a) INFN Gruppo Collegato di Udine, Sezione di Trieste, Udine, Italy; ${ }^{(b)}$ ICTP, Trieste, Italy; ${ }^{(c)}$ Dipartimento Politecnico di Ingegneria e Architettura, Università di Udine, Udine, Italy

68 (a) INFN Sezione di Lecce, Lecce, Italy; ${ }^{(b)}$ Dipartimento di Matematica e Fisica, Università del Salento, Lecce, Italy

69 (a) INFN Sezione di Milano, Milan, Italy; (b) Dipartimento di Fisica, Università di Milano, Milan, Italy

70 (a) INFN Sezione di Napoli, Naples, Italy; ${ }^{(b)}$ Dipartimento di Fisica, Università di Napoli, Naples, Italy

71 (a) INFN Sezione di Pavia, Pavia, Italy; (b) Dipartimento di Fisica, Università di Pavia, Pavia, Italy

72 (a) INFN Sezione di Pisa, Pisa, Italy; (b) Dipartimento di Fisica E. Fermi, Università di Pisa, Pisa, Italy

73 (a) INFN Sezione di Roma, Rome, Italy; ${ }^{(b)}$ Dipartimento di Fisica, Sapienza Università di Roma, Rome, Italy

74 (a) INFN Sezione di Roma Tor Vergata, Rome, Italy; ${ }^{(b)}$ Dipartimento di Fisica, Università di Roma Tor Vergata, Rome, Italy

75 (a) INFN Sezione di Roma Tre, Rome, Italy; ${ }^{(b)}$ Dipartimento di Matematica e Fisica, Università Roma Tre, Rome, Italy

76 (a) INFN-TIFPA, Trento, Italy; ${ }^{(b)}$ Università degli Studi di Trento, Trento, Italy

${ }^{77}$ Institut für Astro- und Teilchenphysik, Leopold-Franzens-Universität, Innsbruck, Austria

78 University of Iowa, Iowa City, IA, USA

${ }^{79}$ Department of Physics and Astronomy, Iowa State University, Ames, IA, USA

${ }^{80}$ Joint Institute for Nuclear Research, Dubna, Russia

81 (a) Departamento de Engenharia Elétrica, Universidade Federal de Juiz de Fora (UFJF), Juiz de Fora,

Brazil; (b) Universidade Federal do Rio De Janeiro COPPE/EE/IF, Rio de Janeiro, Brazil; ${ }^{(c)}$ Instituto de Física,

Universidade de São Paulo, São Paulo, Brazil

${ }^{82}$ KEK, High Energy Accelerator Research Organization, Tsukuba, Japan

${ }^{83}$ Graduate School of Science, Kobe University, Kobe, Japan

84 (a) AGH University of Science and Technology, Faculty of Physics and Applied Computer Science, Kraków, 
Poland; (b) Marian Smoluchowski Institute of Physics, Jagiellonian University, Kraków, Poland

${ }^{85}$ Institute of Nuclear Physics Polish Academy of Sciences, Kraków, Poland

${ }^{86}$ Faculty of Science, Kyoto University, Kyoto, Japan

${ }^{87}$ Kyoto University of Education, Kyoto, Japan

${ }^{88}$ Research Center for Advanced Particle Physics and Department of Physics, Kyushu University, Fukuoka, Japan

${ }^{89}$ Instituto de Física La Plata, Universidad Nacional de La Plata and CONICET, La Plata, Argentina

${ }^{90}$ Physics Department, Lancaster University, Lancaster, UK

${ }^{91}$ Oliver Lodge Laboratory, University of Liverpool, Liverpool, UK

92 Department of Experimental Particle Physics, Jožef Stefan Institute and Department of Physics, University of Ljubljana, Ljubljana, Slovenia

${ }^{93}$ School of Physics and Astronomy, Queen Mary University of London, London, UK

${ }^{94}$ Department of Physics, Royal Holloway University of London, Egham, UK

${ }^{95}$ Department of Physics and Astronomy, University College London, London, UK

${ }^{96}$ Louisiana Tech University, Ruston, LA, USA

${ }^{97}$ Fysiska institutionen, Lunds universitet, Lund, Sweden

${ }^{98}$ Centre de Calcul de l'Institut National de Physique Nucléaire et de Physique des Particules (IN2P3), Villeurbanne, France

${ }^{99}$ Departamento de Física Teorica C-15 and CIAFF, Universidad Autónoma de Madrid, Madrid, Spain

100 Institut für Physik, Universität Mainz, Mainz, Germany

${ }^{101}$ School of Physics and Astronomy, University of Manchester, Manchester, UK

102 CPPM, Aix-Marseille Université, CNRS/IN2P3, Marseille, France

103 Department of Physics, University of Massachusetts, Amherst, MA, USA

${ }^{104}$ Department of Physics, McGill University, Montreal, QC, Canada

105 School of Physics, University of Melbourne, Victoria, Australia

106 Department of Physics, University of Michigan, Ann Arbor, MI, USA

${ }^{107}$ Department of Physics and Astronomy, Michigan State University, East Lansing, MI, USA

108 B.I. Stepanov Institute of Physics, National Academy of Sciences of Belarus, Minsk, Belarus

${ }^{109}$ Research Institute for Nuclear Problems of Byelorussian State University, Minsk, Belarus

${ }^{110}$ Group of Particle Physics, University of Montreal, Montreal, QC, Canada

${ }^{111}$ P.N. Lebedev Physical Institute of the Russian Academy of Sciences, Moscow, Russia

${ }^{112}$ National Research Nuclear University MEPhI, Moscow, Russia

${ }^{113}$ D.V. Skobeltsyn Institute of Nuclear Physics, M.V. Lomonosov Moscow State University, Moscow, Russia

${ }^{114}$ Fakultät für Physik, Ludwig-Maximilians-Universität München, Munich, Germany

115 Max-Planck-Institut für Physik (Werner-Heisenberg-Institut), Munich, Germany

${ }^{116}$ Nagasaki Institute of Applied Science, Nagasaki, Japan

${ }^{117}$ Graduate School of Science and Kobayashi-Maskawa Institute, Nagoya University, Nagoya, Japan

${ }^{118}$ Department of Physics and Astronomy, University of New Mexico, Albuquerque, NM, USA

${ }^{119}$ Institute for Mathematics, Astrophysics and Particle Physics, Radboud University/Nikhef, Nijmegen, The Netherlands

${ }^{120}$ Nikhef National Institute for Subatomic Physics and University of Amsterdam, Amsterdam, The Netherlands

${ }^{121}$ Department of Physics, Northern Illinois University, DeKalb, IL, USA

122 (a) Budker Institute of Nuclear Physics and NSU, SB RAS, Novosibirsk, Russia; (b) Novosibirsk State University

Novosibirsk, Novosibirsk, Russia

${ }^{123}$ Institute for High Energy Physics of the National Research Centre Kurchatov Institute, Protvino, Russia

${ }^{124}$ Institute for Theoretical and Experimental Physics named by A.I. Alikhanov of National Research Centre "Kurchatov Institute", Moscow, Russia

125 Department of Physics, New York University, New York, NY, USA

${ }^{126}$ Ochanomizu University, Otsuka, Bunkyo-ku, Tokyo, Japan

127 Ohio State University, Columbus, OH, USA

${ }^{128}$ Homer L. Dodge Department of Physics and Astronomy, University of Oklahoma, Norman, OK, USA

${ }^{129}$ Department of Physics, Oklahoma State University, Stillwater, OK, USA

${ }^{130}$ RCPTM, Joint Laboratory of Optics, Palacký University, Olomouc, Czech Republic

${ }^{131}$ Institute for Fundamental Science, University of Oregon, Eugene, OR, USA

${ }^{132}$ Graduate School of Science, Osaka University, Osaka, Japan 
133 Department of Physics, University of Oslo, Oslo, Norway

134 Department of Physics, Oxford University, Oxford, UK

${ }^{135}$ LPNHE, CNRS/IN2P3, Sorbonne Université, Université de Paris, Paris, France

136 Department of Physics, University of Pennsylvania, Philadelphia, PA, USA

${ }^{137}$ Konstantinov Nuclear Physics Institute of National Research Centre "Kurchatov Institute", PNPI, St. Petersburg, Russia

${ }^{138}$ Department of Physics and Astronomy, University of Pittsburgh, Pittsburgh, PA, USA

139 (a) Laboratório de Instrumentação e Física Experimental de Partículas-LIP, Lisbon, Portugal; ${ }^{(b)}$ Departamento de Física, Faculdade de Ciências, Universidade de Lisboa, Lisbon, Portugal; ${ }^{(c)}$ Departamento de Física, Universidade de Coimbra, Coimbra, Portugal; ${ }^{(d)}$ Centro de Física Nuclear da Universidade de Lisboa, Lisbon, Portugal; ${ }^{(e)}$ Departamento de Física, Universidade do Minho, Braga, Portugal; ${ }^{(\mathrm{f})}$ Departamento de Física Teórica y del Cosmos, Universidad de Granada, Granada, Spain; ${ }^{(\mathrm{g})}$ Dep Física and CEFITEC of Faculdade de Ciências e Tecnologia, Universidade Nova de Lisboa, Caparica, Portugal; ${ }^{(h)}$ Instituto Superior Técnico, Universidade de Lisboa, Lisbon, Portugal

${ }^{140}$ Institute of Physics of the Czech Academy of Sciences, Prague, Czech Republic

${ }^{141}$ Czech Technical University in Prague, Prague, Czech Republic

${ }^{142}$ Charles University, Faculty of Mathematics and Physics, Prague, Czech Republic

143 Particle Physics Department, Rutherford Appleton Laboratory, Didcot, UK

${ }^{144}$ IRFU, CEA, Université Paris-Saclay, Gif-sur-Yvette, France

${ }^{145}$ Santa Cruz Institute for Particle Physics, University of California Santa Cruz, Santa Cruz, CA, USA

146 (a) Departamento de Física, Pontificia Universidad Católica de Chile, Santiago, Chile; (b) Universidad Andres Bello,

Department of Physics, Santiago, Chile; (c) Instituto de Alta Investigación, Universidad de Tarapacá, Arica,

Chile; ${ }^{(d)}$ Departamento de Física, Universidad Técnica Federico Santa María, Valparaiso, Chile

${ }^{147}$ Universidade Federal de São João del Rei (UFSJ), São João del Rei, Brazil

148 Department of Physics, University of Washington, Seattle, WA, USA

${ }^{149}$ Department of Physics and Astronomy, University of Sheffield, Sheffield, UK

${ }^{150}$ Department of Physics, Shinshu University, Nagano, Japan

${ }^{151}$ Department Physik, Universität Siegen, Siegen, Germany

152 Department of Physics, Simon Fraser University, Burnaby, BC, Canada

${ }^{153}$ SLAC National Accelerator Laboratory, Stanford, CA, USA

${ }^{154}$ Physics Department, Royal Institute of Technology, Stockholm, Sweden

155 Departments of Physics and Astronomy, Stony Brook University, Stony Brook, NY, USA

${ }^{156}$ Department of Physics and Astronomy, University of Sussex, Brighton, UK

${ }^{157}$ School of Physics, University of Sydney, Sydney, Australia

158 Institute of Physics, Academia Sinica, Taipei, Taiwan

159 (a) E. Andronikashvili Institute of Physics, Iv. Javakhishvili Tbilisi State University, Tbilisi, Georgia; ${ }^{(b)}$ High Energy

Physics Institute, Tbilisi State University, Tbilisi, Georgia

${ }^{160}$ Department of Physics, Technion, Israel Institute of Technology, Haifa, Israel

${ }^{161}$ Raymond and Beverly Sackler School of Physics and Astronomy, Tel Aviv University, Tel Aviv, Israel

162 Department of Physics, Aristotle University of Thessaloniki, Thessaloníki, Greece

${ }^{163}$ International Center for Elementary Particle Physics and Department of Physics, University of Tokyo, Tokyo, Japan

${ }^{164}$ Graduate School of Science and Technology, Tokyo Metropolitan University, Tokyo, Japan

165 Department of Physics, Tokyo Institute of Technology, Tokyo, Japan

166 Tomsk State University, Tomsk, Russia

167 Department of Physics, University of Toronto, Toronto, ON, Canada

168 (a) TRIUMF, Vancouver, BC, Canada; ${ }^{(b)}$ Department of Physics and Astronomy, York University, Toronto, ON, Canada

${ }^{169}$ Division of Physics and Tomonaga Center for the History of the Universe, Faculty of Pure and Applied Sciences,

University of Tsukuba, Tsukuba, Japan

${ }^{170}$ Department of Physics and Astronomy, Tufts University, Medford, MA, USA

${ }^{171}$ Department of Physics and Astronomy, University of California Irvine, Irvine, CA, USA

172 Department of Physics and Astronomy, University of Uppsala, Uppsala, Sweden

${ }^{173}$ Department of Physics, University of Illinois, Urbana, IL, USA

${ }^{174}$ Instituto de Física Corpuscular (IFIC), Centro Mixto Universidad de Valencia - CSIC, Valencia, Spain

175 Department of Physics, University of British Columbia, Vancouver, BC, Canada

${ }^{176}$ Department of Physics and Astronomy, University of Victoria, Victoria, BC, Canada 
${ }^{177}$ Fakultät für Physik und Astronomie, Julius-Maximilians-Universität Würzburg, Würzburg, Germany

178 Department of Physics, University of Warwick, Coventry, UK

${ }^{179}$ Waseda University, Tokyo, Japan

${ }^{180}$ Department of Particle Physics and Astrophysics, Weizmann Institute of Science, Rehovot, Israel

${ }^{181}$ Department of Physics, University of Wisconsin, Madison, WI, USA

182 Fakultät für Mathematik und Naturwissenschaften, Fachgruppe Physik, Bergische Universität Wuppertal, Wuppertal, Germany

${ }^{183}$ Department of Physics, Yale University, New Haven, CT, USA

${ }^{a}$ Also at Borough of Manhattan Community College, City University of New York, New York, NY, USA

${ }^{\mathrm{b}}$ Also at Center for High Energy Physics, Peking University, China

${ }^{c}$ Also at Centro Studi e Ricerche Enrico Fermi, Rome, Italy

d Also at CERN, Geneva, Switzerland

e Also at CPPM, Aix-Marseille Université, CNRS/IN2P3, Marseille, France

${ }^{\mathrm{f}}$ Also at Département de Physique Nucléaire et Corpusculaire, Université de Genève, Geneva, Switzerland

g Also at Departament de Fisica de la Universitat Autonoma de Barcelona, Barcelona, Spain

${ }^{\mathrm{h}}$ Also at Department of Financial and Management Engineering, University of the Aegean, Chios, Greece

${ }^{\mathrm{i}}$ Also at Department of Physics and Astronomy, Michigan State University, East Lansing, MI, USA

${ }^{\mathrm{j}}$ Also at Department of Physics and Astronomy, University of Louisville, Louisville, KY, USA

${ }^{k}$ Also at Department of Physics, Ben Gurion University of the Negev, Beer Sheva, Israel

${ }^{1}$ Also at Department of Physics, California State University, East Bay, USA

m Also at Department of Physics, California State University, Fresno, USA

${ }^{\mathrm{n}}$ Also at Department of Physics, California State University, Sacramento, USA

${ }^{\circ}$ Also at Department of Physics, King's College London, London, UK

p Also at Department of Physics, St. Petersburg State Polytechnical University, St. Petersburg, Russia

q Also at Department of Physics, University of Fribourg, Fribourg, Switzerland

${ }^{\mathrm{r}}$ Also at Dipartimento di Matematica, Informatica e Fisica, Università di Udine, Udine, Italy

${ }^{s}$ Also at Faculty of Physics, M.V. Lomonosov Moscow State University, Moscow, Russia

${ }^{\mathrm{t}}$ Also at Giresun University, Faculty of Engineering, Giresun, Turkey

u Also at Graduate School of Science, Osaka University, Osaka, Japan

${ }^{v}$ Also at Hellenic Open University, Patras, Greece

${ }^{w}$ Also at Institucio Catalana de Recerca i Estudis Avancats, ICREA, Barcelona, Spain

x Also at Institut für Experimentalphysik, Universität Hamburg, Hamburg, Germany

y Also at Institute for Nuclear Research and Nuclear Energy (INRNE) of the Bulgarian Academy of Sciences, Sofia, Bulgaria

${ }^{\mathrm{z}}$ Also at Institute for Particle and Nuclear Physics, Wigner Research Centre for Physics, Budapest, Hungary

aa Also at Institute of Particle Physics (IPP), Montreal, Canada

${ }^{\text {ab }}$ Also at Institute of Physics, Azerbaijan Academy of Sciences, Baku, Azerbaijan

ac Also at Instituto de Fisica Teorica, IFT-UAM/CSIC, Madrid, Spain

${ }^{\text {ad }}$ Also at Dept. of Physics, Istanbul University, Istanbul, Turkey

ae Also at Joint Institute for Nuclear Research, Dubna, Russia

${ }^{\text {af }}$ Also at Moscow Institute of Physics and Technology State University, Dolgoprudny, Russia

ag Also at National Research Nuclear University MEPhI, Moscow, Russia

ah Also at Physics Department, An-Najah National University, Nablus, Palestine

ai Also at Physikalisches Institut, Albert-Ludwigs-Universität Freiburg, Freiburg, Germany

aj Also at The City College of New York, New York, NY, USA

${ }^{\text {ak }}$ Also at TRIUMF, Vancouver, BC, Canada

${ }^{\text {al }}$ Also at Universita di Napoli Parthenope, Naples, Italy

${ }^{a m}$ Also at University of Chinese Academy of Sciences (UCAS), Beijing, China

* Deceased 